\title{
ENSAMBLES DE AVES DIURNAS A TRAVÉS DE UN GRADIENTE DE PERTURBACIÓN EN UN PAISAJE EN EL SURESTE DE MÉXICO
}

\author{
NoEl A. GONZÁLEZ-VALDIVIA, ${ }^{1}$ STEFAN L. ARRIAGA-WEISS, ${ }^{2}$ SUSANA \\ OCHOA-GAONA, ${ }^{1 *}$ BRUCE G. FERGUSON, ${ }^{1}$ CHRISTIAN KAMPICHLER ${ }^{2}$ y \\ CARMEN POZO 3 \\ ${ }^{1}$ El Colegio de la Frontera Sur, Sistemas de Producción Alternativos. Apdo. Postal 1042, Admón. \\ de Correos de Tabasco 2000, 86031 Villahermosa, Tabasco, México. Tel/Fax +52 (993) 3136110. \\ <ngonzal@ecosur.mx>; <sochoa@ecosur.mx>; <bferguson@ecosur.mx> \\ 2 División de Ciencias Biológicas. Universidad Juárez Autónoma de Tabasco. Villahermosa, Tabasco, \\ México. <slaw2000@prodigy.net.mx>; <christian.kampichler@web.de> \\ ${ }^{3}$ El Colegio de la Frontera Sur. Conservación de la Biodiversidad, Chetumal, Quintana Roo, México. \\ <cpozo@ecosur.mx> \\ * Autor para correspondencia.
}

González-Valdivia N. A., S. L. Arriaga-Weiss, S. Ochoa-Gaona, B. G. Ferguson, C. Kampichler \& C. Pozo. 2012. Ensambles de aves diurnas a través de un gradiente de perturbación en un paisaje en el sureste de México. Acta Zoológica Mexicana (n. s.), 28(2): 237-269.

RESUMEN. Con el objetivo de evaluar la diversidad de aves que se presentan en un paisaje del trópico mexicano, en el año 2008 se evaluó la riqueza, abundancia y distribución de las aves y sus gremios de forrajeo en ocho unidades del paisaje agrupadas en dos ecomosaicos. De las 218 especies identificadas, 207 estuvieron presentes en la estación seca y 119 en la de lluvias. Se encontró que la composición de avifauna difiere entre los ecomosaicos (a) matriz agropecuaria heterogénea y (b) bosque tropical perennifolio, así como entre las unidades de paisaje anidadas en ellos. La riqueza varió de 76 a 118 especies entre unidades del paisaje, mientras que el índice de diversidad de Shannon-Wiener resultó similar (de 3.20 a 4.44), y las comunidades de aves se distribuyeron equitativamente (Pielou: 0.71 a 0.91). En el paisaje se configura un escenario complementario entre ecomosaicos y una diversidad de aves muy alta, incluyendo especies importantes para la conservación como Tinamus major, Crypturellus soui, Crax rubra, Amazona farinosa, Odontophorus guttatus, Chondrohierax uncinatus, Platyrinchus cancrominus, Onycorhynchus coronatus y Manacus candei. Los gremios de forrajeo de aves se separan en función de las características ambientales de las unidades de paisaje por ejemplo, en los potreros con árboles dispersos predominan cazadoras desde percha y especies asociadas al ganado, mientras que en el bosque predominan los insectívoros que buscan sus presas en el suelo, corteza y follaje. Algunas especies como Lipaugus unirufus, Pipra mentalis y Schiffornis turdinus pueden funcionar como indicadoras ecológicas del bosque conservado, mientras otras como Sporophila americana, S. torqueola, Pitangus sulphuratus,

Recibido: 07/05/2010; aceptado: 08/02/2012. 
Myozetetes similis, Cyanocorax morio y Crotophaga sulcirostris lo son para el estado perturbado del bosque nativo. El paisaje actual conformado por remanentes de bosque y una matriz agropecuaria con arbolado, posibilita la conservación biológica de aves, constituyendo un modelo potencial para su refugio y manejo sustentable. Sin embargo, la expansión de la ganadería extensiva, el abandono del sistema de rotación milpa-acahual y la extracción gradual de madera del bosque en el ejido, pone en riesgo la diversidad de la avifauna en la zona de estudio.

Palabras clave: bosque tropical, diversidad de aves, gremios de forrajeo, ecología del paisaje, heterogeneidad.

González-Valdivia N. A., S. L. Arriaga-Weiss, S. Ochoa-Gaona, B. G. Ferguson, C. Kampichler \& C. Pozo. 2012. Diurnal bird assemblages along a disturbance gradient in a landscape in Southeast Mexico. Acta Zoológica Mexicana (n. s.), 28(2): 237-269.

ABSTRACT. In order to assess of a Mexican tropical landscape like birds shelter, in the 2008 we evaluated richness, abundance, distribution and foraging guilds of birds in eight landscape units, nested within two ecomosaics. Of 218 species identified, 207 were recorded during dry season and 119 in the rain season. Species composition differed between the two ecomosaics, (a) heterogeneous agriculture matrix and (b) rain forest remnant, and among their nested landscape units. Shannon-Wiener index was similar (from 3.20 to 4.44) among landscape units, and mean species richness varied from 76 to 118 species, while avian communities were equally distributed (Pielou: 0.71 to 0.91 ). At the landscape scale, the two ecomosaics play complementary roles in maintaining high bird diversity, providing habitat for high value conservation species like Tinamus major, Crypturellus soui, Crax rubra, Amazona farinosa, Odonthophorus guttatus, Chondrohierax uncinatus, Platyrinchus cancrominus, Onycorhynchus coronatus and Manacus candei. Foraging guilds frequencies differ by landscape units. For example in pasturelands with spare trees, perch hunters and cattle-associated birds were abundant, while insectivores gleaning from soil, bark and foliage were common into the forest. Species like Lipaugus unirufus, Pipra mentalis and Shiffornis turdinus could be ecological indicators of conserved forest conditions while Sporophila americana, S. torqueola, Pitangus sulphuratus, Cyanocorax morio and Crotophaga sulcirostris favor disturbed native forest habitat. Current patterns of landscape management constitute a potential model of sustainable management for both agricultural production and bird conservation. However this management model is threatened by external and internal drivers of extensive cattle ranching, the abandonment of swidden systems and gradual extraction of wood from local forests.

Key words: tropical forest, birds’ diversity, landscape ecology, heterogeneity, foraging guilds

\section{INTRODUCCIÓN}

Los elementos característicos de un paisaje son la matriz que corresponde a la cobertura dominante — en nuestra zona de estudio las áreas agropecuarias_-, dentro de la cual se incluyen los fragmentos de vegetación natural, en este caso la selva o bosque tropical (Forman \& Godron 1981, Turner et al. 2001). Un paisaje cultural está dominado y modelado por la actividad humana (Higgs 2003). Desde la perspectiva de Luginbühl (1998; citado por Álvarez-Areces 2009), éste se concibe como el reconocimiento de formas visibles de prácticas agrícolas, de una estructura paisajística particular, así como una particular localización y forma de los árboles, de los campos de cultivo y el relieve. Si en el paisaje así definido, se conserva una gran diversidad — en este caso de aves_- 
de manera complementaria entre bosques remanentes y áreas agrícolas, se estaría en presencia de un modelo potencial de manejo local ecológicamente sustentable.

El estudio del efecto de las perturbaciones humanas sobre las comunidades biológicas, en particular la avifauna neotropical, ha sido abordado por diferentes autores (Andrade \& Rubio-Torgler 1994, Daily et al. 2001, Cárdenas et al. 2003, Harvey \& González-Villalobos 2007, Cerezo et al. 2009). Anteriormente, la conservación de la avifauna se enfocaba principalmente a ecosistemas naturales, los bosques, dejando relegados los ambientes agropecuarios (Kupfer et al. 2006). Esta tendencia, ha cambiado a partir de los 90's en los que se ha resaltado el papel de la matriz como complemento para la conservación (Estrada et al. 1997, Beier \& Noss 1998, Perfecto \& Vandermeer 2002, Driscoll 2005; Harvey et al. 2005, Schröth \& Harvey 2007; Martin et al. 2009; Pulido-Santacruz \& Renjifo 2011). Entonces, parece posible en un paisaje cultural, compatibilizar la producción con la conservación de la biodiversidad preservando suficientes áreas boscosas naturales que se conecten mediante matrices agropecuarias heterogéneas (Kupfer et al. 2006, Castellón \& Sievig 2006, Vandermeer \& Perfecto 2007). Estas últimas, deben incluir diversos arreglos de árboles como componentes del manejo de las parcelas (Estrada et al. 1997, Schröth \& Harvey 2007, Perfecto \& Vandermeer 2008). En México, aun cuando existen inventarios de especies de aves para numerosas áreas, las investigaciones que analizan las relaciones entre componentes bióticos con todos los elementos del paisaje aún son escasas (Almazán-Núñez et al. 2009), a pesar de la importancia que esta información tiene para orientar el manejo sustentable de los recursos naturales (Bell et al. 1997).

Las características del paisaje influyen en la composición y abundancia de las aves, pudiendo facilitar o impedir el mantenimiento de algunas especies (Gillespie \& Walter 2001), por lo que este grupo ha sido considerado útil para evaluar la calidad del hábitat (Da Silva et al. 1996, Fleishman et al. 2002, Gray et al. 2007, Arriaga-Weiss et al. 2008). Lo anterior es especialmente importante cuando se evalúa la matriz agropecuaria como hábitat utilizado por especies y los diferentes gremios de forrajeo de la avifauna nativa (Stouffer et al. 2006; Arriaga-Weiss et al. 2008).

El objetivo de este estudio es evaluar la diversidad de aves que se presentan en el paisaje del ejido Niños Héroes de Chapultepec, Tenosique, Tabasco como representativo del trópico mexicano, mediante la comparación cuantitativa de la riqueza, abundancia y distribución de las especies de aves, así como entre sus gremios de forrajeo como grupos funcionales considerando la estacionalidad al incluir muestreos en la época seca y la época lluviosa. En este artículo se identificó y comparó la avifauna nativa del bosque con la presente en las unidades de la matriz agropecuaria. Mediante correlaciones y análisis canónico se examinó la influencia de las características estructurales y físico-ambientales de las unidades del paisaje sobre la avifauna y sus gremios. 


\section{MATERIAL Y MÉTODOS}

Área de estudio. La zona se ubica dentro del ejido Niños Héroes de Chapultepec (en adelante "ejido"), municipio de Tenosique, Tabasco, en el sureste de México

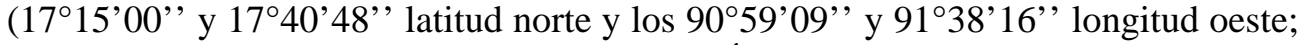
Ochoa-Gaona et al. 2008). El ejido es parte del Área Protegida Cañón del Usumacinta (DOF 2008). El relieve es de valles entre montañas abarcando una superficie de 2066 ha. El clima es cálido y húmedo con una corta estación seca, presentando $26{ }^{\circ} \mathrm{C}$ promedio de temperatura anual y 2750 mm de precipitación media anual (Isaac-Márquez et al. 2005); la altitud fluctúa entre 200 y 700 msnm.

La vegetación original es el bosque tropical perennifolio, dominado por Manilkara zapota, Dialium guianense, Terminalia amazonia, Brosimum alicastrum y Aspidosperma megalocarpon en el dosel, con un estrato más bajo en el que destacan Sebastiania tuerkheimanii y Alchornea latifolia. El sotobosque está dominado por Psychotria spp., Faramea occidentalis, Rinorea guatemalensis y Cryosophila argentea; el estrato herbáceo por Pteridophyta, Araceae y Zingiberaceae (GonzálezValdivia 2010). Del bosque se extrajo cedro (Cedrela odorata) y caoba (Swietenia macrophylla) en la década de 1970 (Gobierno del Estado de Tabasco 1997), lo que produjo los primeros indicios de perturbación a estos bosques. La reserva ejidal de bosque es parte del manejo del territorio por la comunidad humana establecida en él, razón que motivó la selección del ejido Niños Héroes de Chapultepec como área para realizar el estudio desde la perspectiva del paisaje cultural.

Ecomosaicos y unidades de paisaje. Se diferenciaron dos ecomosaicos, que de acuerdo con Rossignol et al. (1998) corresponden a un mosaico de unidades de vegetación que pertenecen a un mismo ambiente. Los ecomosaicos diferenciados fueron: la matriz agropecuaria y el bosque tropical perennifolio. Este último, es considerado dentro del paisaje del ejido como el hábitat de referencia, así como su avifauna, y sirve de base como punto de comparación (para todos los índices) y como una medida de la calidad como refugio ecológico de la avifauna en ese paisaje. Se considera como refugio ecológico al potencial que uno o un conjunto de ambientes que tienen para proporcionar albergue, conectividad, alimento u otros recursos del ámbito de hogar que permitan parcial o totalmente la permanencia y persistencia de una o varias especies de la avifauna nativa o migratoria naturalmente asociada al sitio (Olson et al. 2002). En especial, éste enfoque sobre la condición de manejo resulta útil al permitir contrastar la matriz agropecuaria, como ecomosaico que concentra el mayor impacto humano respecto al ecosistema original, pero sin negar la complementariedad de ambos espacios como parte del potencial de conservación de la biodiversidad en el paisaje.

Las unidades de paisaje (sensu Zonneveld 1989) fueron identificadas en recorridos in situ. En la matriz agropecuaria se diferenciaron cuatro unidades: a) vegetación secundaria madura con desarrollo $>15$ años, en este texto llamados acahuales maduros, b) vegetación secundaria 5-7 años o acahuales jóvenes, c) árboles en línea 
en potreros formando cercos vivos y d) potreros con árboles dispersos cuya función principal es suministrar sombra o forraje al ganado. En el ecomosaico de bosque tropical perennifolio como el sitio de referencia, se consideraron las siguientes unidades que seguían la posición de las unidades de la matriz por el patrón de su localización en ese ecomosaico: a) bosque en las partes altas de las montañas como referente de la vegetación secundaria madura, b) bosque en las laderas como referente de la vegetación secundaria joven, c) bosque en las partes planas como referente de los pastizales con árboles dispersos y d) elementos de perturbación lineal dentro del bosque por senderos de acceso. Cada una de las cuatro réplicas que se hicieron por unidad fue muestreada mediante tres puntos de conteo visitados cuatro veces por estación (Fig. 1). Los muestreos se hicieron durante el año 2008, tanto en la época seca como en la lluviosa. Se caracterizó ambiental y estructuralmente cada unidad (Cuadro 1).

Muestreo. Se aplicó el método de puntos de conteo con radio fijo sugerido por Hutto et al. (1986) y Bibby et al. (2000). Se establecieron 12 puntos de conteo con radio fijo de $25 \mathrm{~m}$ para cada unidad (tres puntos por cada una de las cuatro réplicas que se establecieron por cada unidad), separados por al menos $200 \mathrm{~m}$ de distancia. La observación diaria se hizo de 6:00 a 9:00 AM durante diez minutos en cada punto, contando a todos los individuos por especie, vistos y escuchados, dentro del radio establecido. Cada punto fue visitado una vez cada semana por cuatro semanas consecutivas, para obtener así un total de 48 conteos por unidad del paisaje en la estación seca (de febrero a abril) y 48 en la estación lluviosa (de julio a septiembre) del año 2008.

Las variables ambientales temperatura y humedad relativa fueron medidas dentro de cada punto de conteo por cada repetición de unidad utilizando HOBO Data Logger colocados a una altura aproximada de un metro y en un radio de dos metros del centro del punto de conteo. De manera similar fueron determinadas la pendiente mediante clinómetro Suunto y la insolación por fotografías hemisféricas (Rich et al. 1999). Para caracterizar la estructura de la vegetación de las unidades de paisaje, se establecieron doce parcelas permanentes de $500 \mathrm{~m}^{2}$ cada una. Dentro de estas parcelas se obtuvo el área basal midiendo el diámetro a la altura del pecho de los árboles (d.a.p. $\geq 5 \mathrm{~cm}$ ). La altura del dosel se calculó con clinómetro y la cobertura del dosel con un densitómetro. El número de estratos observado por unidad se registró con base en cuatro categorías: arbolado alto $(\geq 15 \mathrm{~m}$ ), arbolado bajo ( $\geq 5 \mathrm{~m} \mathrm{y}<15 \mathrm{~m}$ ), sotobosque arbustivo y sotobosque herbáceo.

Grupo de estudio. La identificación de las especies, familias y sus gremios de forrajeo se hizo cotejando con las guías ilustradas de Howell \& Webb (1995) y Van Perlo (2006), así como registros grabados de sus cantos respecto a la Biblioteca de Sonidos Aves de México (González-García 2002). Se identificó el gremio de forrajeo que expresa un nivel de funcionalidad ecológica de cada especie con base en Robinson (2001) y Gillespie (2002). Los 16 gremios de forrajeo resultantes y sus códigos fueron: cazador acuático (5 especies), cazador o insectívoro asociado a ga- 


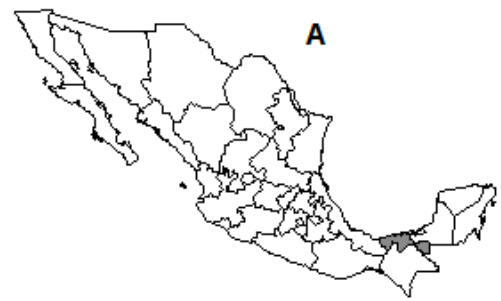

B

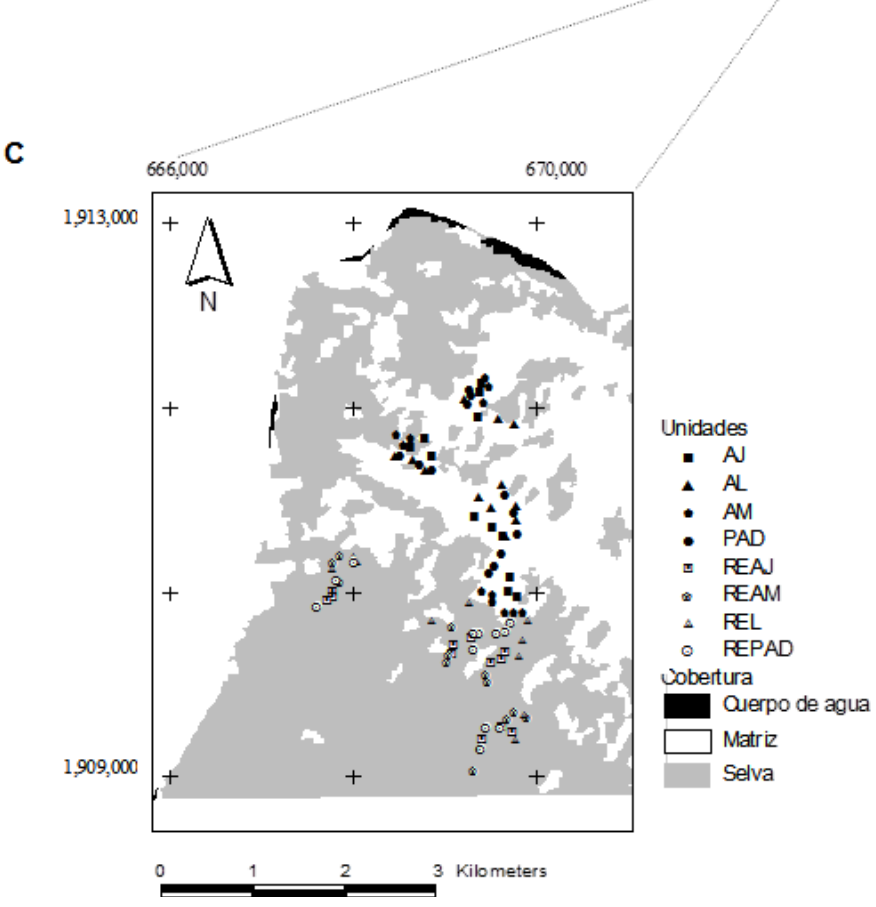

Figura 1. Sitio de estudio y puntos de conteo establecidos en el bosque y la matriz agropecuaria del ejido Niños Héroes de Chapultepec, Tenosique, Tabasco. A) Tabasco, México, B) Municipio de Tenosique y C) Ejido. Código: $\mathrm{RE}$ = bosque, $\mathrm{RE}_{\mathrm{AJ}}=$ bosque en laderas referente de vegetación secundaria joven, $\mathrm{RE}_{\mathrm{AM}}$ = bosque en las parte altas de montañas referente de vegetación secundaria madura, $\mathrm{RE}_{\mathrm{PAD}}=$ bosque en las partes planas referente de potreros con árboles dispersos, $\mathrm{REL}=$ senderos dentro del bosque, $\mathrm{MA}$ = matriz agropecuaria, $\mathrm{AJ}$ = acahual o vegetación secundaria joven, $\mathrm{AM}=$ acahual o vegetación secundaria madura, $\mathrm{AL}$ = árboles en línea formando cercos vivos, $\mathrm{PAD}=$ potrero con árboles dispersos.

nado (2 especies), carroñero (2 especies), cazador que captura presas sobre corteza o en epifitas (8 especies), cazador que captura presas dentro de la madera (carpintero; 7 especies), granívoro-frugívoro arbóreo (18 especies), granívoro-frugívoro terrestre (24 especies), insectívoro pequeño que caza sin percha en el estrato alto y medio (38 


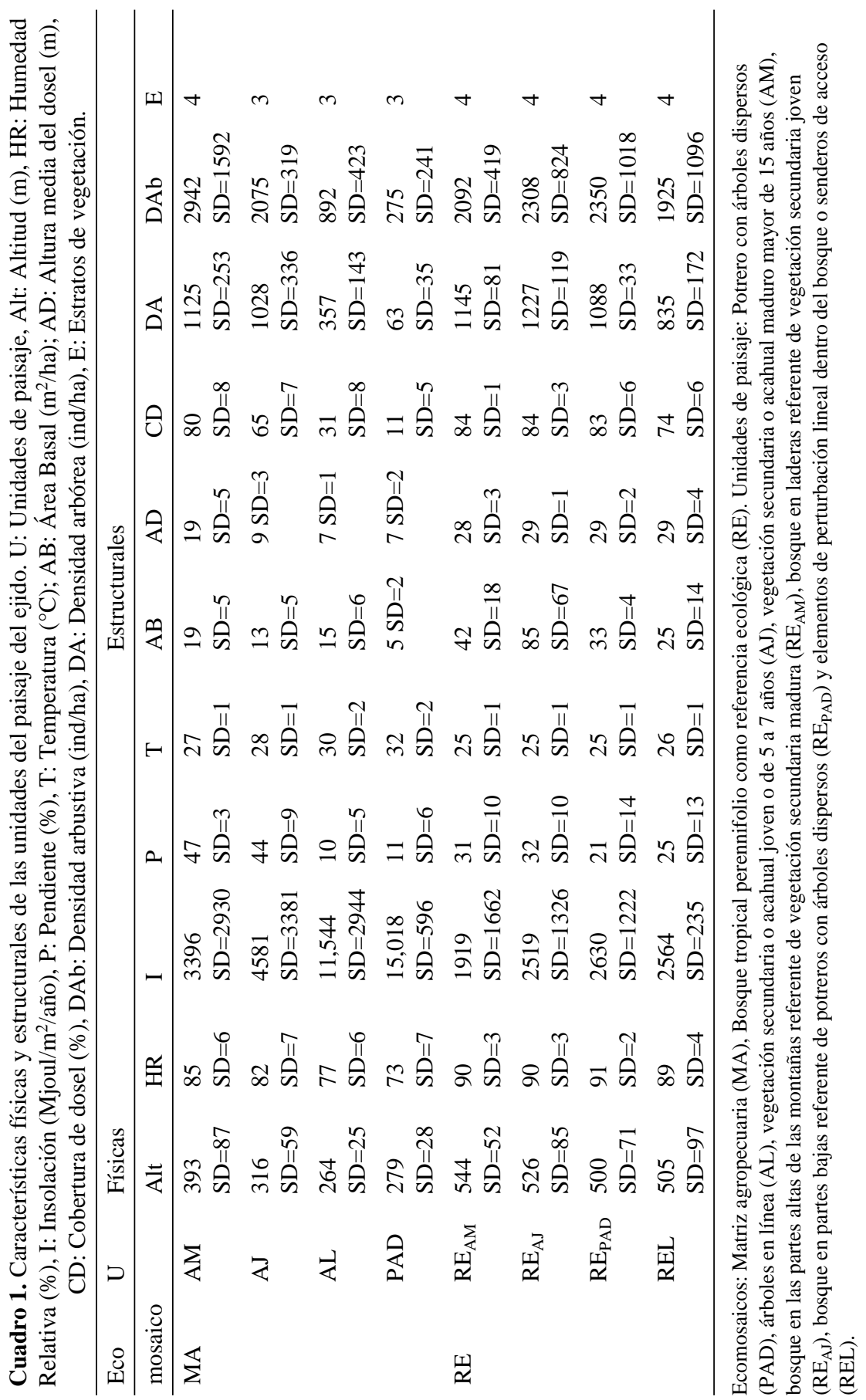


especies), insectívoro pequeño que caza sin percha en el estrato arbustivo bajo y herbáceo (26 especies), insectívoro grande cazador bajo el dosel desde percha $(\geq 15 \mathrm{~cm}$ longitud; 15 especies); insectívoro pequeño cazador bajo el dosel desde percha ( $<15$ cm longitud; 23 especies), insectívoro en el suelo (13 especies), insectívoro que caza al vuelo sobre el dosel (golondrina) (1 especie), nectarívoro (18 especies), omnívoros arbóreos (4 especies), omnívoros (5 especies), rapaz diurna (9 especies).

Eficiencia de muestreo. Utilizando el número de observaciones por especie en cada unidad del paisaje, se trazaron curvas de acumulación mediante el índice de rarefacción o de Coleman (EstimateS 7.5.2, Colwell 2006). Con base en la ecuación de Clench (1979), se determinó el valor esperado de especies contra el cual se compararon las riquezas observadas y con este procedimiento se evaluó la eficiencia del esfuerzo de muestreo. Se asumió que si ésta resultaba $\geq 80 \%$ del ensamble esperado, el muestreo fue representativo.

Diversidad alfa. Se calcularon los índices de diversidad, dominancia y de equitatividad de los ensambles de aves presentes en cada unidad (Pérez et al. 2007), utilizando PAST 2.0 (Hammer et al. 2001). Para establecer si había efectos debidos a la unidad, ecomosaicos y estaciones del año en el número de especies registradas y sus abundancias medias estandarizadas (Hutto et al. 1986), se hizo análisis de varianza de una vía (ANDEVA, $\alpha=0.05$ ). Se exploró la existencia de relaciones entre las variables estructurales y físicas respecto a las abundancias medias estandarizadas y las riquezas de especies por cada unidad de paisaje mediante la correlación de Pearson $(\alpha=0.05)$ que permite relacionar adimensionalmente las variables cuantitativas. Para hacer esto se comprobaron los supuestos de normalidad y homocedasticidad en la distribución de los datos de las variables de estudio apoyados en el programa SPSS 10.0. (Morrison 1999).

Diversidad beta. Se exploraron los datos mediante la prueba de Box \& Cox (1964) y aplicando la Ley de Taylor (Herrando-Pérez 2002) para identificar si requerían transformación previa a los análisis multivariados. Se aplicó el análisis por cúmulos con el método de agrupamiento por pares de medias no ponderadas (UPGMA) usando como distancia al índice Bray-Curtis (Zuur et al. 2007) para determinar agrupamientos de la avifauna por estación climática y unidades de paisaje y obtener la magnitud de las similitudes o disimilitudes entre ensambles de cada unidad de paisaje respecto a las demás. Se determinó cómo influyen las características ambientales de las unidades del paisaje en los ensambles de especies y gremios de forrajeo de aves utilizando análisis de correspondencia canónica, con CANOCO 5.0 (Lepš \& Šmilauer 2005). La identificación de especies indicadoras de unidades de la matriz agropecuaria y del bosque se realizó mediante el análisis de similitudes porcentuales (SIMilarity PERcentage) en PRIMER 6.0 (Clarke \& Gorley 2006) tomando como criterio para elegir a estas especies cuando aparecieran dentro del grupo de aves que aportaban el 50\% de similitud dentro de una unidad de paisaje y además que apare- 
cieran en más de una de las unidades pertenecientes a un ecomosaico, como aproximación a la fidelidad al bosque o a la matriz agropecuaria.

\section{RESULTADOS}

Eficiencia de muestreo. En el campo se observaron 5,221 individuos correspondientes a 218 especies de aves agrupadas en 33 familias que representan el 95\% de las 230 especies esperadas para el paisaje. En la matriz se observaron el $90 \%$ y en el bosque el 89\% del total esperado respectivamente (Fig. 2).

Diversidad alfa. En las unidades del paisaje se obtuvieron registros desde 76 especies en los árboles en línea hasta 118 especies en las unidades de bosque. Los índices ecológicos muestran comunidades equitativamente distribuidas, con diversidad de media a alta según el índice de Shannon-Wiener $\left(\mathrm{H}^{\prime}>3.20\right)$ y sin que se observe dominancia de una especie sobre las demás, según el índice Simpson (D < 0.09; Cuadro 2).

Se encontraron diferencias en el número de especies observadas para todo el paisaje en las dos estaciones de muestreo $(\mathrm{F}(\mathrm{n}=2, \mathrm{r}=8, \mathrm{gl}=1)=130.9, p<0.001)$, pero no respecto al número medio de individuos totales registrados $(\mathrm{F}(\mathrm{n}=2, \mathrm{r}=8, \mathrm{gl}=1)$ $=0.616, p=0.446)$ entre la estación seca (93 \pm 10 especies y 2 individuos avistados en promedio por punto de conteo y la lluviosa (40 \pm 9 especies y 2 individuos avistados en promedio). De las 218 especies registradas en total, 130 fueron residentes, 65 especies migratorias y 23 aparentemente migraron de manera local (Cuadro 3). En la época seca se registraron con exclusividad a 98 especies, mientras que en la lluviosa se registraron 10 especies. A cada una de éstas cifras se deben agregar las 109 especies presentes en ambas épocas, totalizando así 207 especies registradas en la estación seca y 119 en la de lluvias respectivamente. Por otro lado, en la época de lluvias se reportó como exclusiva a una especie migratoria mientras que 38 lo fueron para la época seca. El total de especies migrantes locales estuvo representado en la temporada de secas (21 especies) mientras seis especies resultaron migrantes locales en la época de lluvias. Cuatro de estas migrantes locales estuvieron presentes en ambas épocas. La migración local, probablemente en búsqueda de alimentos, puede dirigirse hacia el interior del bosque continuo de El Petén, Guatemala que está adyacente al territorio ejidal. No hubo reporte de especies migrantes locales exclusivas de la época lluviosa (Cuadro 3). Si consideramos a la avifauna total que utilizó las unidades de paisaje, éstas no difieren en la riqueza de especies $(\mathrm{F}(\mathrm{n}=8, \mathrm{r}=4, \mathrm{gl}=7)=0.36, p=0.923)$ que fluctuó entre 26 y 34 especies observadas por recuento, ni en el número medio de individuos registrados para cada especie $(\mathrm{F}(\mathrm{n}=8, \mathrm{r}=4, \mathrm{gl}=7)=0.043, p=1.000)$.

Diversidad beta. De las 218 especies identificadas, 170 se registraron en el bosque tropical perennifolio y 173 en la matriz agropecuaria (144 en las unidades de 


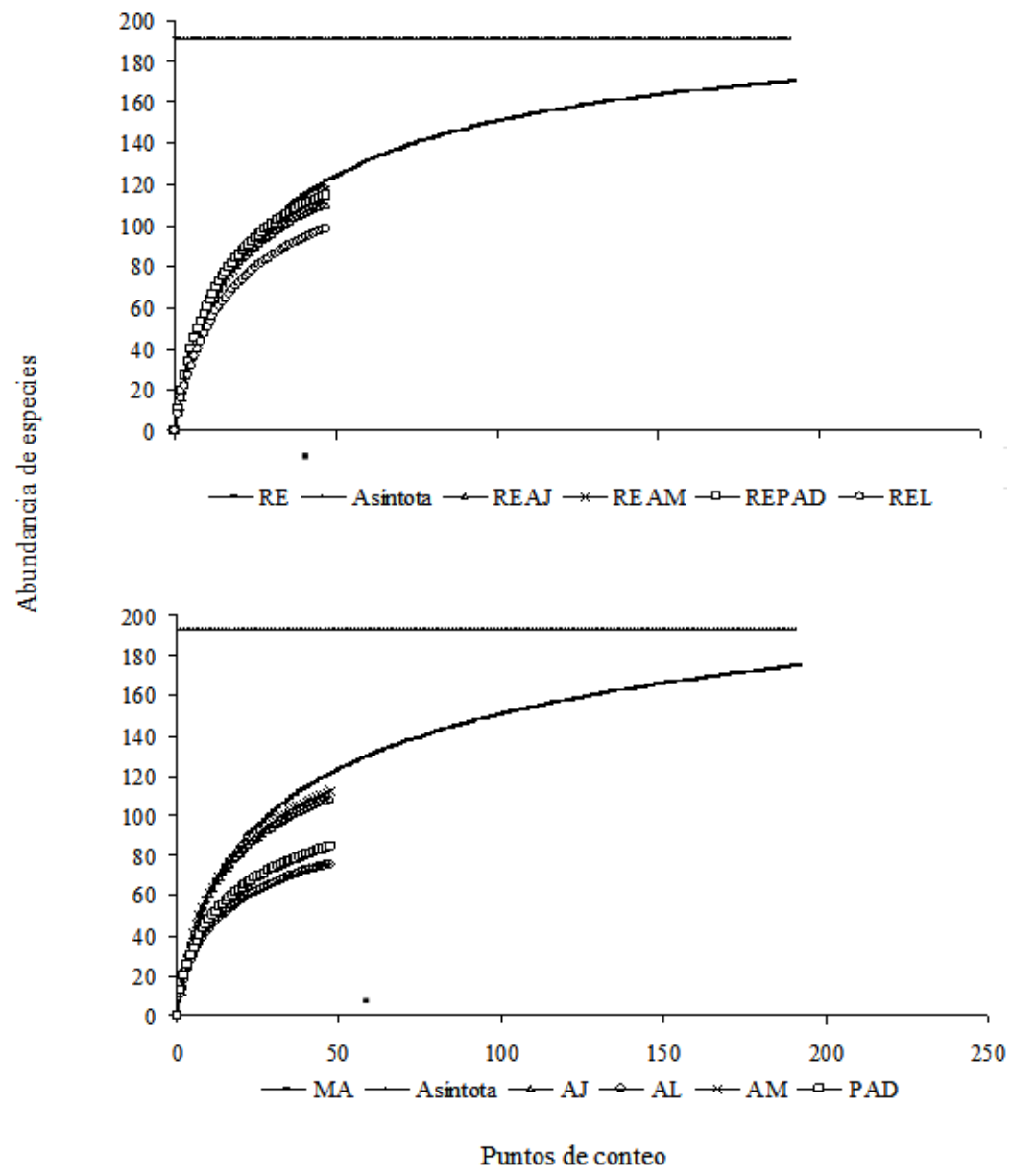

Figura 2. Acumulación de especies de aves para el ecomosaico RE (superior), MA (inferior) y unidades de paisaje. Asíntota = 230 spp. Código: $\mathrm{RE}=$ bosque, $\mathrm{RE}_{\mathrm{AJ}}=$ bosque en laderas referente de vegetación secundaria joven, $\mathrm{RE}_{\mathrm{AM}}$ = bosque en las partes altas de montañas referente de vegetación secundaria madura, $\mathrm{RE}_{\mathrm{PAD}}$ = bosque en las partes planas referente de potreros con árboles dispersos, $\mathrm{REL}$ = senderos dentro del bosque, $\mathrm{MA}=$ matriz agropecuaria, $\mathrm{AJ}=$ acahual o vegetación secundaria joven, $\mathrm{AM}$ = acahual o vegetación secundaria madura, $\mathrm{AL}$ = árboles en línea formando cercos vivos, $\mathrm{PAD}=$ potrero con árboles dispersos.

vegetación secundaria y 107 especies en los potreros con árboles dispersos y en los cercos vivos). De las especies reportadas en el paisaje, 126 (58\%) son compartidas entre el bosque y la matriz agropecuaria. La diferencia entre ensambles se debe a 44 especies reportadas únicamente en el bosque (20\%) y 48 especies sólo en la matriz agropecuaria (22\%; Anexo 1). Este cúmulo boscoso es similar en cerca del $30 \%$ al 
Cuadro 2. Índices de diversidad para las unidades de paisaje del ejido A) Total, B) Estación seca y C) Estación lluviosa. Unidades de paisaje: Potrero con árboles dispersos (PAD), árboles en línea $(A L)$, vegetación secundaria o acahual joven $(A J)$, vegetación secundaria o acahual maduro (AM), bosque en las partes altas de las montañas referente de vegetación secundaria madura $\left(\mathrm{RE}_{\mathrm{AM}}\right)$, bosque en laderas referente de vegetación secundaria joven $\left(R E_{A J}\right)$, bosque en partes bajas referente de potreros con árboles dispersos $\left(\mathrm{RE}_{\mathrm{PAD}}\right)$ y elementos de perturbación lineal dentro del bosque o senderos de acceso (REL).

\begin{tabular}{lllllllll}
\hline A) Total & PAD & $\mathrm{AL}$ & $\mathrm{AJ}$ & $\mathrm{AM}$ & $\mathrm{REL}$ & $\mathrm{RE}_{\mathrm{AM}}$ & $\mathrm{RE}_{\mathrm{AJ}}$ & $\mathrm{RE}_{\mathrm{PAD}}$ \\
\hline Especies observadas & 85 & 76 & 109 & 112 & 101 & 118 & 112 & 115 \\
Especies esperadas & 104 & 94 & 135 & 142 & 133 & 166 & 142 & 151 \\
Individuos registrados & $1077^{\mathrm{a}}$ & $693^{\mathrm{b}}$ & $743^{\mathrm{b}}$ & $659^{\mathrm{b}}$ & $449^{\mathrm{b}}$ & $469^{\mathrm{b}}$ & $561^{\mathrm{b}}$ & $571^{\mathrm{b}}$ \\
Dominancia D & 0.09 & 0.05 & 0.03 & 0.02 & 0.03 & 0.02 & 0.02 & 0.02 \\
Diversidad H' & 3.20 & 3.53 & 4.10 & 4.30 & 4.19 & 4.44 & 4.28 & 4.30 \\
Equitatividad J & 0.71 & 0.82 & 0.87 & 0.91 & 0.91 & 0.93 & 0.91 & 0.91 \\
\hline
\end{tabular}

\begin{tabular}{lllllllll}
\hline B) Secas & $\mathrm{PAD}$ & $\mathrm{AL}$ & $\mathrm{AJ}$ & $\mathrm{AM}$ & $\mathrm{REL}$ & $\mathrm{RE}_{\mathrm{AM}}$ & $\mathrm{RE}_{\mathrm{AJ}}$ & $\mathrm{RE}_{\mathrm{PAD}}$ \\
\hline Especies observadas & 78 & 72 & 95 & 100 & 91 & 102 & 101 & 107 \\
Individuos registrados & 725 & 518 & 533 & 490 & 332 & 278 & 410 & 378 \\
Dominancia D & 0.08 & 0.05 & 0.02 & 0.02 & 0.03 & 0.02 & 0.02 & 0.02 \\
Diversidad H' & 3.29 & 3.57 & 4.09 & 4.12 & 4.07 & 4.35 & 4.19 & 4.23 \\
Equitatividad J & 0.76 & 0.84 & 0.90 & 0.90 & 0.90 & 0.94 & 0.91 & 0.93 \\
\hline
\end{tabular}

\begin{tabular}{lllllllll}
\hline C) Lluvias & $\mathrm{PAD}$ & $\mathrm{AL}$ & $\mathrm{AJ}$ & $\mathrm{AM}$ & $\mathrm{REL}$ & $\mathrm{RE}_{\mathrm{AM}}$ & $\mathrm{RE}_{\mathrm{AJ}}$ & $\mathrm{RE}_{\mathrm{PAD}}$ \\
\hline Especies observadas & 29 & 30 & 47 & 49 & 31 & 46 & 47 & 46 \\
Individuos registrados & 352 & 175 & 210 & 168 & 117 & 191 & 151 & 193 \\
Dominancia D & 0.15 & 0.09 & 0.05 & 0.04 & 0.06 & 0.04 & 0.04 & 0.04 \\
Diversidad H' & 2.40 & 2.79 & 3.38 & 3.54 & 3.15 & 3.50 & 3.50 & 3.44 \\
Equitatividad J & 0.71 & 0.82 & 0.88 & 0.91 & 0.92 & 0.92 & 0.91 & 0.90 \\
\hline
\end{tabular}

formado por las unidades de vegetación secundaria y ambos resultan totalmente separados del grupo conformado por la avifauna de potreros con árboles en línea y árboles dispersos (Fig. 3). El aporte de las especies de aves migratorias y de las residentes a la separación de unidades tiene un valor de importancia semejante entre ambos grupos (Fig.4) y mayor que el que aportan las migrantes locales estacionales. No se encontraron correlaciones entre las variables estructurales y físico-ambientales respecto a la riqueza de especies y sus abundancias. Por otro lado, el análisis de correspondencia canónica aplicado a los gremios de forrajeo de avifauna entre las unidades de paisaje detectó que los gremios resultan influidos en su preferencia de hábitat por variables 
Cuadro 3. Distribución de las especies de aves según su estatus migratorio y estación climática en la que se registraron en las unidades del paisaje del ejido.

\begin{tabular}{lcccccccccc}
\hline Estación & Estatus & \multicolumn{8}{c}{ Unidades de paisaje } \\
\hline \multirow{4}{*}{ Seca } & & PAD & AL & AJ & AM & REL & REAM & REAJ & REPAD & Total \\
& MIG & 33 & 32 & 36 & 35 & 26 & 29 & 32 & 38 & 64 \\
& MIE & 4 & 1 & 7 & 9 & 8 & 14 & 11 & 12 & 21 \\
& RES & 41 & 39 & 52 & 56 & 57 & 59 & 58 & 57 & 122 \\
& Total & $\mathbf{7 8}$ & $\mathbf{7 2}$ & $\mathbf{9 5}$ & $\mathbf{1 0 0}$ & $\mathbf{9 1}$ & $\mathbf{1 0 2}$ & $\mathbf{1 0 1}$ & $\mathbf{1 0 7}$ & $\mathbf{2 0 7}$ \\
\multirow{5}{*}{ Lluvias } & MIG & 6 & 7 & 6 & 8 & 5 & 5 & 7 & 9 & 24 \\
& MIE & 1 & 2 & 1 & 2 & 0 & 1 & 1 & 2 & 6 \\
& RES & 22 & 21 & 40 & 39 & 26 & 40 & 39 & 35 & 89 \\
& Total & $\mathbf{2 9}$ & $\mathbf{3 0}$ & $\mathbf{4 7}$ & $\mathbf{4 9}$ & $\mathbf{3 1}$ & $\mathbf{4 6}$ & $\mathbf{4 7}$ & $\mathbf{4 6}$ & $\mathbf{1 1 9}$ \\
& MIG & 34 & 32 & 37 & 37 & 28 & 32 & 35 & 33 & 65 \\
& MIE & 5 & 3 & 8 & 9 & 8 & 15 & 11 & 13 & 23 \\
& RES & 46 & 41 & 64 & 66 & 65 & 71 & 66 & 69 & 130 \\
& Total & 85 & 76 & 109 & 112 & 101 & 118 & 112 & 115 & 218 \\
\hline
\end{tabular}

Código: MIG = Migrante, MIE = Migrante local estacional, RES $=$ Residente.

Unidades de paisaje: Potrero con árboles dispersos (PAD), árboles en línea (AL), vegetación secundaria o acahual joven (AJ), vegetación secundaria o acahual maduro (AM), bosque en las partes altas de las montañas referente de vegetación secundaria madura $\left(R E_{A M}\right)$, bosque en laderas referente de vegetación secundaria joven $\left(R E_{A J}\right)$, bosque en partes bajas referente de potreros con árboles dispersos $\left(\mathrm{RE}_{\mathrm{PAD}}\right)$ y elementos de perturbación lineal dentro del bosque o senderos de acceso (REL).

físicas como la temperatura, la humedad relativa y la altitud así como por las variables estructurales de cobertura y altura media del dosel (Fig. 5).

Especies indicadoras. Se identificaron especies y familias indicadoras de las unidades y ecomosaicos del paisaje. Dentro de los hábitats más perturbados, potreros con árboles en línea y con árboles dispersos, las especies que más aportaron en similitud fueron los insectívoros grandes a medianos que cazan desde perchas (Pitangus sulphuratus, Tyrannus melancholicus) y los especialistas de hábitats abiertos como Crotophaga sulcirostris, Cyanocorax morio, Dives dives o Sporophila spp. (Cuadro 4). Las familias que más aportan a la similitud entre unidades y ecomosaicos del paisaje fueron Emberizidae, Troglodytidae y Tyrannidae (acumulan 50\% de similitud), mientras las que aportan más a la diferencia resultan ser Trochilidae y Psittacidae ( $\geq 30 \%$, Cuadro 5). Para el bosque las especies características fueron Ramphastos sulfuratus, Lipaugus unirufus, Pipra mentalis, Schiffornis turdinus, Rhytipterna holerythra, Xiphorhynchus flavigaster, Uropsila leucogastra, Amazona farinosa, Leptotila cassinni y Trogon massena (Cuadro 4). Mientras las familias más importantes para caracterizar este ecomosaicos fueron Ramphastidae, Cotingidae y Psittacidae (Cuadro 5). 


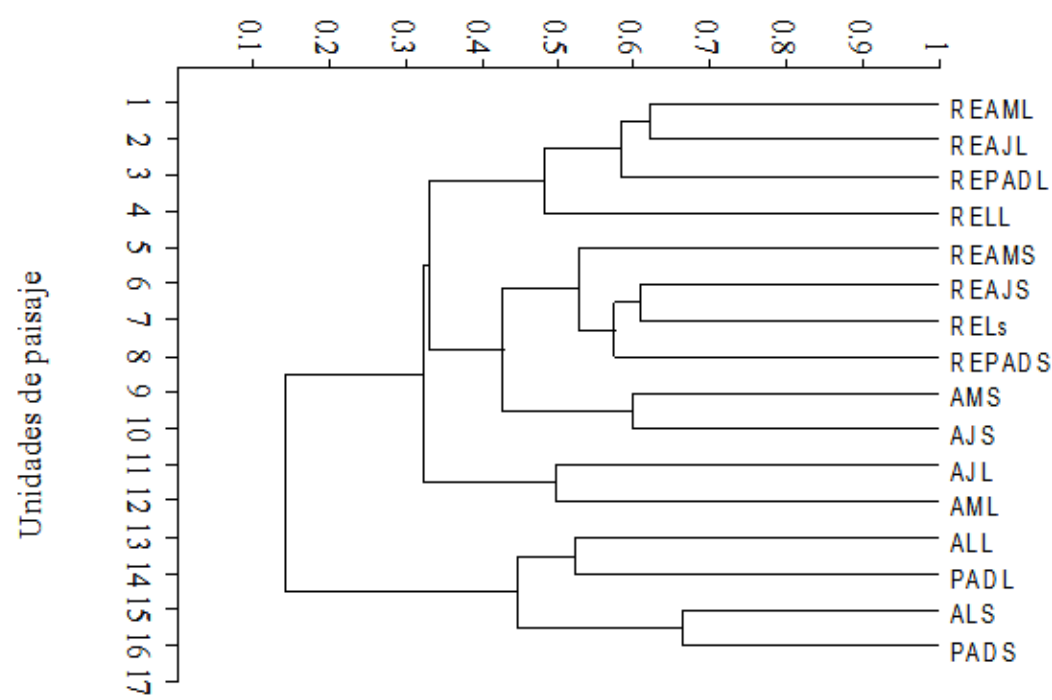

Figura 3. Dendrograma para unidades de paisaje y estaciones cuando se muestreó la avifauna. bosque en las partes altas de las montañas referente de vegetación secundaria madura $\left(\mathrm{RE}_{\mathrm{AM}}\right)$, bosque en laderas referente de vegetación secundaria joven $\left(\mathrm{RE}_{\mathrm{AJ}}\right)$, bosque en las partes planas referente de potreros con árboles dispersos $\left(\mathrm{RE}_{\mathrm{PAD}}\right)$ y senderos dentro del bosque (REL), vegetación secundaria o acahual joven (AJ), vegetación secundaria o acahual maduro (AM), árboles en línea formando cercos vivos (AL), potrero con árboles dispersos (PAD).

\section{DISCUSIÓN}

Diversidad alfa. Los ensambles de aves difieren por efecto del manejo ejidal. En coincidencia con Farina (1997), la matriz resulta el ecomosaico más rico en especies, probablemente debido a la diversidad estructural que le proporciona la combinación de las cuatro unidades de paisaje que contiene, en particular las de vegetación secundaria en dos estados sucesionales. Por otra parte, un gran número de especies del bosque encuentran refugio en la vegetación secundaria incrementando su riqueza. En las áreas abiertas (potreros) se favorece a las especies insectívoras y granívoras. Los estanques o abrevaderos atraen aves acuáticas que no frecuentan el bosque ni los acahuales incrementando la riqueza en aves en los potreros. La complementariedad de la riqueza de especies entre unidades de bosque tropical conservado y unidades de la matriz agropecuaria puede ser una posible evidencia del efecto de disturbio intermedio propuesto por Connell (1978). Este efecto establece que aquellos ecosistemas sujetos a perturbaciones con frecuencia moderada permiten la mayor diversidad toda vez que bajo ese tipo de régimen las poblaciones de la comunidad pueden recuperarse bien entre episodios de perturbación y la apertura temporal de nuevos nichos permiten que otras especies puedan utilizarlos ampliándose por esas dos vías la riqueza y diversidad de especies que conviven en la comunidad. Las unidades de vegetación 

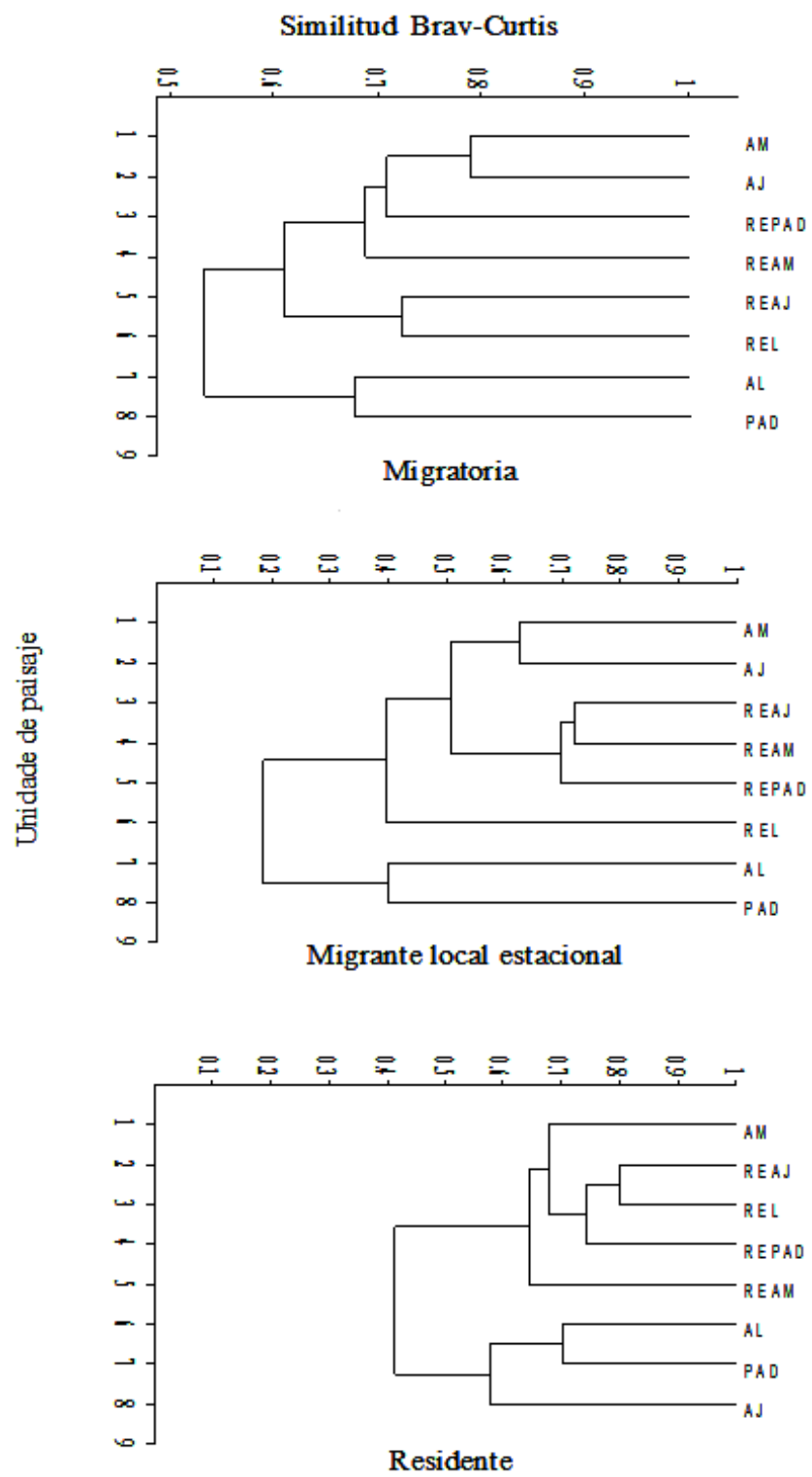

Figura 4. Dendrogramas de especies de aves migratorias (Arriba), migrantes locales estacionales (Medio) y residentes (Abajo) y su aporte a los ensambles de las unidades de paisaje. bosque en las partes altas de las montañas referente de vegetación secundaria madura $\left(\mathrm{RE}_{\mathrm{AM}}\right)$, bosque en laderas referente de vegetación secundaria joven $\left(\mathrm{RE}_{\mathrm{AJ}}\right)$, bosque en las partes planas referente de potreros con árboles dispersos (RE $\mathrm{RAD}_{\mathrm{PAD}}$ ) y senderos dentro del bosque (REL), vegetación secundaria o acahual joven (AJ), vegetación secundaria o acahual maduro (AM), árboles en línea formando cercos vivos (AL), potrero con árboles dispersos (PAD), Estaciones: $\mathrm{S}=$ Seca, $\mathrm{L}=$ lluviosa. 


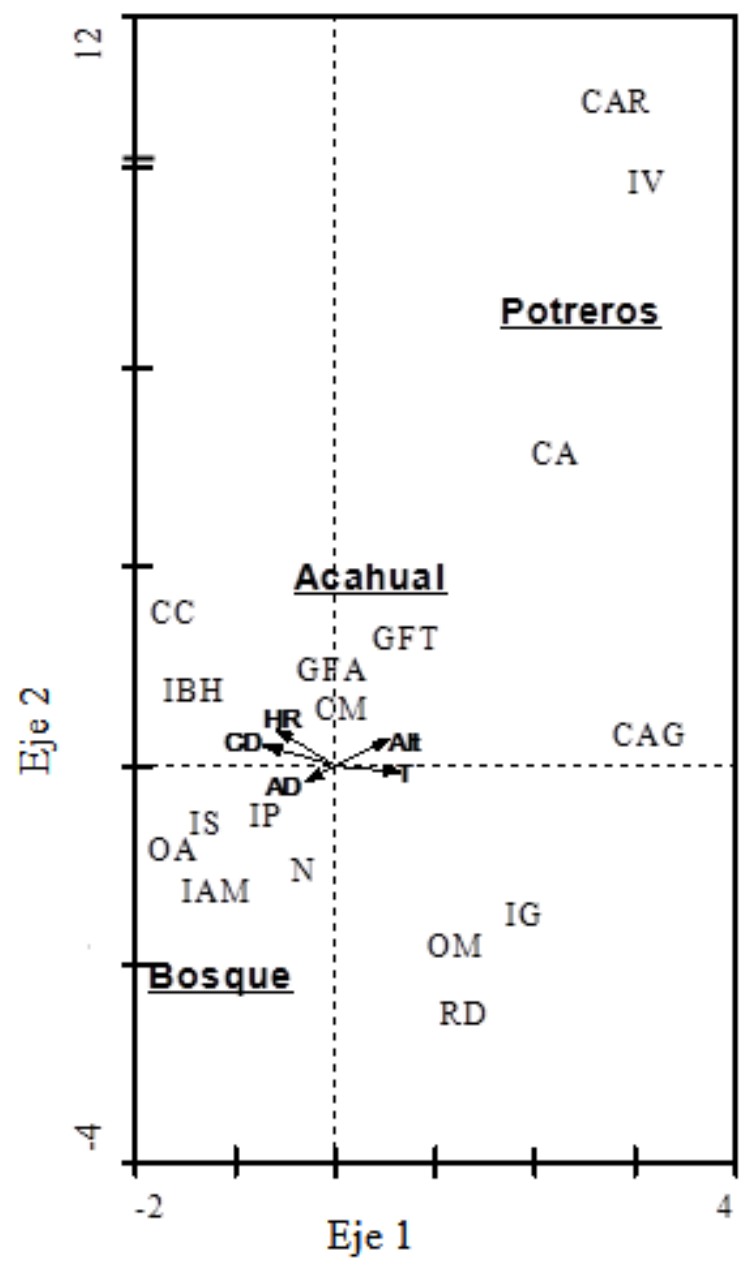

Figura 5. Correlación canónica para gremios de forrajeo de aves en el ejido. Gremios: CA = Cazador acuático, CAG = Cazador asociado a ganado, CAR = Carroñero, $\mathrm{CC}=$ Cazador que captura presas sobre corteza o en epifitas, $\mathrm{CM}=$ Cazador que captura presa dentro de la madera (carpintero), GFA = Granívoro-frugívoro arbóreo, GFT = Granívoro-frugívoro terrestre, IAM = Insectívoro pequeño que caza sin percha en el estrato alto y medio, IBH = Insectívoro pequeño que caza sin percha en el estrato arbustivo bajo y herbáceo, IG = Insectívoro grande cazador bajo el dosel desde percha ( $\geq 15 \mathrm{~cm}$ longitud), IP = Insectívoro pequeño cazador bajo el dosel desde percha (15 cm longitud), IS = Insectívoro en el suelo, IV = Insectívoro que caza al vuelo sobre el dosel, $\mathrm{N}=$ Nectarívoro, OA = Omnívoros arbóreo, $\mathrm{OM}=$ Omnívoros, $\mathrm{RD}=$ Rapaz diurna. Variables ambientales: $\mathrm{AD}=$ Altura del dosel, Alt $=$ Altitud $(\mathrm{m}), \mathrm{CD}=$ Cobertura del dosel, $\mathrm{HR}=$ Humedad relativa $(\%)$ y $\mathrm{T}=$ Temperatura $\left({ }^{\circ} \mathrm{C}\right)$. 
Cuadro 4. Aves más relevantes para identidad de unidades del paisaje en NHC. Potrero con árboles dispersos (PAD), árboles en línea (AL), vegetación secundaria joven (AJ), vegetación secundaria madura (AM), bosque en la parte alta de montañas referente de vegetación secundaria madura $\left(\mathrm{RE}_{\mathrm{AM}}\right)$, bosque en laderas referente de vegetación secundaria joven $\left(\mathrm{RE}_{\mathrm{AJ}}\right)$, bosque en las partes planas referente de pastizales con árboles dispersos $\left(\mathrm{RE}_{\mathrm{PAD}}\right)$ y perturbación lineal o senderos de acceso dentro del bosque (REL). Los números representan el aporte porcentual de las especies a la similitud de cada unidad.

\begin{tabular}{|c|c|c|c|c|c|c|c|c|}
\hline \multirow[b]{3}{*}{ Especie/Unidad de paisaje } & \multicolumn{8}{|c|}{ Ecomosaico } \\
\hline & \multicolumn{4}{|c|}{ Matriz agropecuaria (MA) } & \multicolumn{4}{|c|}{ Remanente de bosque (RE) } \\
\hline & PAD & $\mathrm{AL}$ & AJ & $\mathrm{AM}$ & $\mathrm{RE}_{\mathrm{AM}}$ & $\mathrm{RE}_{\mathrm{AJ}}$ & $\mathrm{RE}_{\mathrm{PAD}}$ & REL \\
\hline Tyrannus melancholicus & 4.90 & 4.42 & 1.72 & & & & & \\
\hline Sporophila americana & 7.81 & 5.90 & 1.54 & & & & & \\
\hline Sporophila torqueola & 6.75 & 5.18 & 1.95 & & & & & \\
\hline Dives dives & 4.07 & 4.15 & 3.51 & & & & & \\
\hline Pitangus sulfuratus & 4.23 & 4.25 & 1.48 & & & & & \\
\hline Columbina minuta & 4.04 & 4.68 & 3.81 & & & & & \\
\hline Crotophaga sulcirostris & 5.38 & 5.88 & 1.99 & & & & & \\
\hline Cyanocorax morio & 4.30 & 4.33 & 5.36 & 3.87 & & & & \\
\hline Leptotila verreauxi & & & 3.83 & 2.90 & & & & \\
\hline Trogon citreolus & & & 0.61 & 3.78 & 0.69 & 0.57 & 3.57 & \\
\hline Ramphocaenus melanurus & & & 4.59 & 4.73 & 2.10 & 4.48 & 4.78 & 5.14 \\
\hline Troglodytes musculus & & & 5.49 & 4.44 & 4.52 & 4.43 & 4.41 & 5.44 \\
\hline Henicorhina leucosticta & & & & 3.45 & 2.37 & 1.82 & 2.27 & 2.16 \\
\hline Trogon melanocephalus & & & & 1.77 & 4.08 & 3.63 & 1.94 & 4.23 \\
\hline Ramphastos sulfuratus & & & & 3.07 & 4.60 & 4.05 & 4.09 & 4.23 \\
\hline Lipaugus unirufus & & & & 1.96 & 5.76 & 4.89 & 5.58 & 6.42 \\
\hline Pipra mentalis & & & & & 1.88 & 3.87 & 3.81 & 0.80 \\
\hline Schiffornis turdinus & & & & & 2.11 & 3.29 & 3.63 & 1.89 \\
\hline Rhitiptherna holerithra & & & & & 4.36 & 0.74 & 0.59 & 0.87 \\
\hline Xiphorhynchus flavigaster & & & & & 3.88 & 1.53 & 1.96 & 0.73 \\
\hline Urocoptila leucogastra & & & & & 4.61 & 1.99 & 2.03 & \\
\hline Amazona farinosa & & & & & 2.10 & 2.26 & 0.59 & \\
\hline Leptotila cassini & & & & & 3.74 & 0.59 & & \\
\hline Trogon massena & & & & & & 3.20 & 1.77 & 0.77 \\
\hline
\end{tabular}

secundaria resultado del cese de actividades de cultivo y el descanso del suelo, actividades propias del manejo del sistema milpa, promueven una riqueza de especies muy alta en la matriz agropecuaria del paisaje estudiado. La riqueza de especies de aves registrada dentro del bosque tropical (170 especies) fue similar a la reportada por Estrada et al. (1997) en los Tuxtlas y por Ramírez-Albores (2010) en la Depresión Central de Chiapas, siendo mayor el número de especies que reportamos en este 
Cuadro 5. Familias de aves que contribuyen en la identidad de las unidades de paisaje en NHC.

Potrero con árboles dispersos (PAD), árboles en línea formando cercos vivos (AL), vegetación secundaria o acahual joven (AJ), vegetación secundaria madura o acahual maduro (AM), bosque en partes altas de las montañas referente de vegetación secundaria madura $\left(\mathrm{RE}_{\mathrm{AM}}\right)$, bosque en laderas referente de vegetación secundaria joven $\left(\mathrm{RE}_{\mathrm{AJ}}\right)$, bosque en las partes planas referente de potreros con árboles dispersos ( $\left.\mathrm{RE}_{\mathrm{PAD}}\right) \mathrm{y}$, perturbación lineal o senderos de acceso dentro del bosque (REL). Las cifras reflejan el aporte porcentual de las familias a la similitud de cada unidad.

\begin{tabular}{|c|c|c|c|c|c|c|c|c|}
\hline \multirow[b]{3}{*}{ Familia } & \multicolumn{8}{|c|}{ Ecomosaico } \\
\hline & \multicolumn{4}{|c|}{ Matriz agropecuaria (MA) } & \multicolumn{4}{|c|}{ Remanente de bosque (RE) } \\
\hline & PAD & $\mathrm{AL}$ & $\mathrm{AJ}$ & $\mathrm{AM}$ & $\mathrm{RE}_{\mathrm{AM}}$ & $\mathrm{RE}_{\mathrm{AJ}}$ & $\mathrm{RE}_{\mathrm{PAD}}$ & REL \\
\hline Corvidae & 8.4 & 6.8 & 10.7 & 2.97 & & & & \\
\hline Emberizidae & 23.7 & 20.2 & 13.4 & 11.3 & 7.2 & 5.9 & 11.9 & 17.1 \\
\hline Sylviidae & & & 4.7 & 5.0 & & 6.9 & 7.5 & 5.6 \\
\hline Trogonidae & & & 6.0 & 9.4 & 7.0 & 8.7 & 9.7 & 3.9 \\
\hline Troglodytidae & & & 12.3 & 12.3 & 15.8 & 11.3 & 12.9 & 16.8 \\
\hline Ramphastidae & & & & 2.8 & 6.9 & 4.5 & 4.5 & 3.9 \\
\hline Cotingidae & & & & & 13.5 & 11.1 & 11.9 & 10.8 \\
\hline Psittasidae & & & & & 2.5 & 3.1 & 3.5 & \\
\hline
\end{tabular}

trabajo para este hábitat a lo registrado por otros autores (Cuadro 6). La riqueza total en el ejido (218 especies) es ligeramente menor que las 225 especies reportadas en Chiapas. Los registros de avifauna en las diferentes unidades del paisaje del ejido al ser comparados con otros estudios del neotrópico muestran valores similares en riqueza (Cuadro 6). Los índices de diversidad en las unidades del paisaje en nuestro estudio $\left(3.20 \leq \mathrm{H}^{\prime} \leq 4.44\right)$ superaron a los reportados por Ramírez-Albores $(2010)$ en Chiapas (máximo valor del índice Shannon $\mathrm{H}^{\prime}=3.39$ ) y en general representaron una alta riqueza (sensu Ramírez 2006). Incluso la avifauna asociada a los potreros con árboles dispersos $\left(\mathrm{H}^{\prime}=3.20\right)$ y en árboles en línea $\left(\mathrm{H}^{\prime}=3.53\right)$ fue alta alojando en conjunto al $49 \%$ de la avifauna del ejido, proporción similar a la reportada por Ramírez-Albores (2010) en los potreros que estudió en Chiapas.

En el bosque se encontró que la perturbación lineal ocasionada por los senderos de acceso a su interior disminuyó la riqueza de especies (99 especies) respecto al bosque no perturbado (de 112 a 118 especies), esto coincide con lo encontrado por Develey \& Stouffer (2001) en bosques de la Amazonía afectados por caminos.

Las diferencias en la composición de especies se deben a las modificaciones del hábitat de bosque, consecuencia de la actividad agropecuaria. Los cambios tienden a simplificar la estructura al suprimir los árboles del dosel y favorecer a los árboles pioneros de porte más bajo, aumentando el espaciamiento entre un árbol y otro, disminuyendo así la cobertura y densidad forestal. 
Cuadro 6. Síntesis de algunas investigaciones sobre avifauna en agropaisajes y bosques húmedos tropicales. Los números dentro del cuadro indican la riqueza de especies de aves registrada en la unidad de paisaje. $\mathrm{AL}$ = cerca viva/árboles en línea, $\mathrm{PAD}=$ potrero con árboles dispersos, $\mathrm{AJ}=$ vegetación secundaria joven (5 a 7 años), AM = vegetación secundaria madura (15 a 20 años), CA = cultivos anuales, $\mathrm{CP}=$ cultivos permanentes, $\mathrm{RE}=$ Bosque.

\begin{tabular}{|c|c|c|c|c|c|c|c|}
\hline \multicolumn{8}{|c|}{ Agropaisaje } \\
\hline Autor & $\mathrm{AL}$ & PAD & $\mathrm{AJ}$ & $\mathrm{AM}$ & CA & $\mathrm{CP}$ & $\mathrm{RE}$ \\
\hline $\mathrm{a}$ & & & & & & & 157 \\
\hline b & & & & & & & 125 \\
\hline c & & & 54 & $50-52$ & & & \\
\hline d & & 32 & & 41 & & & 20 \\
\hline e & 42 & $28-45$ & 45 & & & & $30-39$ \\
\hline $\mathrm{f}$ & 89 & 70 & & & & & 134 \\
\hline g & 97 & 12 & & & $9-31$ & $65-123$ & 178 \\
\hline $\mathrm{h}$ & 75 & & & & & & \\
\hline $\mathrm{i}$ & & 73 & & & & & \\
\hline $\mathrm{j}$ & & & & & & & 158 \\
\hline $\mathrm{k}$ & & & & & & 84 & \\
\hline l & & & & & & & 350 \\
\hline $\mathrm{m}$ & 92 & & & & & & \\
\hline $\mathrm{n}$ & 76 & 51 & 128 & 128 & 54 & & $69-178$ \\
\hline Ejido & 76 & 85 & 109 & 113 & & & 170 \\
\hline
\end{tabular}

a. Anderson 2010, b. Arriaga-Weiss et al. (2008), c. Bojorges-Baños y López-Mata (2001), d. Bojorges-Baños y López-Mata (2006), e. Cárdenas et al. (2003), f. Cerezo et al. (2009), g. Estrada et al. (1997), h. Francesconi (2006), i. Guevara y Laborde (1993), j. Hueck-Espino (2005), k. Ibarra et al. (2001), l. Patten et al. (2010), m. Lang et al. (2003), n. Ramírez-Albores (2010), Ejido = Nuestro estudio.

Se encontró que la composición y riqueza de la avifauna cambió entre estaciones, con mayor riqueza en la época seca tal como otros autores encontraron en diferentes tipos de vegetación (Estrada et al. 1997, MacNally et al. 2002). Esto se debe a la migración tanto local como estacional. La primera fue observada al menos en dos especies de Psittacidae (Amazona farinosa y Aratinga holochlora) y de Trochilidae (Anthracothorax prevostii y Chlorostilbon canivetii) que reaparecieron al final de la época de lluvias después de estar ausentes buena parte de esa estación o como se presentó con Tyrannidae con 15 especies ausentes durante el periodo de lluvias pero abundantes en la época de secas.

Diversidad beta. La correspondencia canónica entre grupos de aves según su gremio de forrajeo respecto a los distintos hábitats comprobó el impacto del manejo sobre la composición de los ensambles en el paisaje y distinguió el ensamble de aves 
presente en las unidades del bosque de aquel presente en las unidades de la matriz agropecuaria. Se mostró que algunas especies y familias prefieren un tipo de hábitat particular, tal como se ha observado en otros estudios (Fleishman et al. 2002). El patrón de ensambles se mantuvo incluso entre la estación seca y la lluviosa. En ambos casos los Tyrannidae (Tyrannus spp., Pitangus sulphuratus o Myiarchus spp.) junto a especies como Crotophaga sulcirostris o Turdus grayi resultan estrechamente asociados a ecosistemas abiertos (potreros). Otras como Schiffornis turdinus y Lipaugus unirufus especies cazadoras del sotobosque o Formicarius nigricapillus cazadora sobre la superficie del suelo y la hojarasca, están asociadas a la mayor altura del dosel y del número de estratos de vegetación, así como a temperaturas menores bajo el dosel del bosque y de acahuales maduros (Vielliard 2000, Hill \& Hamer 2004; Watling \& Donnelly (2006).

La matriz agropecuaria del ejido es capaz de sostener comunidades importantes de aves debido principalmente a su diversidad estructural (Tews et al. 2004; Atauri \& De Lucio 2001; Cerezo et al. 2009). En el paisaje, las riquezas y abundancias de aves, son muy próximas entre los dos ecomosaicos debido a la presencia de los acahuales de la matriz que funcionan como hábitats intermedios entre los contrastes de los pastizales y el bosque tropical perennifolio. Esto puede ser considerado una potencial expresión del efecto de disturbio intermedio de Connell (1978) debido a que la vegetación secundaria dentro del sistema de milpa del ejido constituye una fase que permite un cese de la perturbación agrícola por un tiempo medio de 15 a 20 (30) años. Las unidades de vegetación secundaria o acahuales de la matriz agropecuaria del ejido bajo estudio, permitieron una riqueza de aves similar a la del bosque, aunque con diferente composición (similar a Andrade \& Rubio-Torgler 1994).

Entre los ecomosaicos del paisaje, no hubo diferencias en cuanto a riqueza pero si en la composición de especies, demostrando de esta manera la existencia de impactos del manejo en la avifauna. Sin embargo, el actual ensamble biológico del ejido podría ser afectado aún más debido a un impacto drástico (sensu Fletcher \& Hutto 2008) como la ampliación de potreros en todo el paisaje que es la principal amenaza para las selvas o bosques tropicales de esta región (Isaac-Márquez et al. 2005).

La sobrevivencia de varias especies de aves parece depender fuertemente de grandes fragmentos de bosques protegidos (Castelleta et al. 2000); este es el caso de Crax rubra y Xiphorhynchus flavigaster habitantes de los remanentes de bosque del ejido. Además, las características propias de la matriz agropecuaria y su diversidad estructural también brindan refugio y alimentos a una cantidad importante de aves (Lehouk et al. 2009). De hecho, en la zona de estudio, la avifauna presenta algunas especies características de la matriz y otras del bosque. Esto se extendió a los gremios de forrajeo de manera similar a lo reportado en un bosque tropical perturbado (Gray et al. 2007). Los resultados difieren de los de Daily et al. (2001) quienes encontraron que en un paisaje costarricense conformado por dos ecomosaicos, hábitats cerrados (selva 
o bosque tropical) y hábitats abiertos (matriz agropecuaria), 149 (55\%) de las 272 especies de aves identificadas eran características exclusivas del bosque, mientras solamente un $23 \%$ (63 especies) eran propias de la matriz, existiendo un $20 \%$ de aves compartidas por ambos ecomosaicos (60 especies). En el caso del paisaje tabasqueño estudiado, la avifauna compartida ocupa una proporción mayor (126 especies, 58\%), mientras que 44 especies (20\%) fueron exclusivamente características del bosque y 48 especies (22\%) resultaron características de la matriz. Las variaciones pudieron deberse a diferencias en las matrices y bosques o a la composición de la avifauna entre ambos sitios, pues la diversidad biológica se distribuye, como lo afirman RangelSalazar et al. (2009) de manera heterogénea en la geografía terrestre.

Las aves del bosque estuvieron bien representadas en la reserva del ejido destacando los gremios picamaderos (Xiphorhynchus flavigaster, Xenops minutus, Sittasomus griseicapillus), omnívoro arbóreo (Trogon massena), insectívoro pequeño sin percha (Pipra mentalis), nectarívoro (Trochilidae), insectívoro en el suelo u hojarasca (Henicorhina spp., Thryothorus spp., Microcerculus philomela) o insectívoro pequeño cazador desde percha (Myiopagis viridicata, Mitrephanes phaeocercus, Onychorhynchus coronatus, Terenotriccus erythrurus, Tyranniscus vilissimus). No obstante, se detectaron gremios con especies aparentemente especialistas de bosque (ArriagaWeiss et al. 2008), como los carpinteros (Campephilus guatemalensis, Dryocopus lineatus, Piculus rubiginosus), granívoros-frugívoros terrestres (Ortalis vetula) y granívoros-frugívoros arbóreos (Psittacidae) que tuvieron buena representación en la vegetación secundaria (Fig. 5).

Los omnívoros (Cyanocorax morio), rapaces (Accipritidae, Falconidae) e insectívoros grandes cazadores desde percha (Myiarchus spp., Tyrannus spp.) predominaron, junto a los cazadores acuáticos y carroñeros en las unidades abiertas (potreros y líneas de árboles). En el caso de las rapaces, los resultados contrastan con lo encontrado en un bosque tropical lluvioso en Tabasco, donde se registró una mayor presencia del gremio en grandes fragmentos de bosque (Arriaga-Weiss et al. 2008), mientras en nuestro estudio frecuentaron la matriz agropecuaria. Una probable causa es que en los hábitats abiertos se mejoran las probabilidades de capturar presas para ciertas especies (Bildstein \& Zalles 2001; Filloy \& Bellocq 2007). También algunas rapaces como Chondrohierax uncinatus y Buteo magnirostris fueron avistadas en la época seca, contrario a lo reportado por Vázquez-Pérez et al. (2009) quienes en la Reserva de la Biósfera Selva El Ocote, Chiapas, observaron una mayor frecuencia de estas aves en la época de lluvias, pero de manera similar, la mayor riqueza de rapaces se presentó en las áreas más perturbadas (zona de amortiguamiento). Por otro lado, la presencia de árboles aislados o en línea permite que aves de los gremios granívorosfrugívoros terrestres, omnívoros y tiránidos grandes se refugien en los potreros, tal como han encontrado otros autores (Martin et al. 2009), además que se favoreció la presencia de rapaces ya que se usan estos árboles para descansar o cazar desde allí. 
El reemplazo o la sustitución de especies pertenecientes a las mismas familias o gremios debido a los cambios de hábitat conservados hacia alterados y abiertos tiene ejemplo en el ejido con Trochilidae. En este caso las especies de bosque Campylopterus rufus, Eupherusa eximia y Threnetes ruckeri encontradas en la reserva de bosque dan lugar en acahuales jóvenes y potreros con árboles en línea o dispersos a aquellas especies propias de sabanas como Heliomaster longirostris, Amazilia tzacatl, A. yucatanensis y Chlorostilbon canivetii.

El efecto de refugio debido a la heterogeneidad matricial, principalmente por la persistente práctica tradicional del descanso prolongado de la tierra en el paisaje del ejido y lo arbolado de los potreros resulta complementario a los ecomosaicos de bosque (Shankar-Raman et al. 1998; Castellón \& Sieving 2006). Incluso especies que han sido extirpadas de paisajes similares como Crax rubra o Pachyramphus cinnamomeus (Patten et al. 2010), existen en el ejido aparentemente por estar diversificado ambientalmente, lo que le transfiere su valor como refugio de aves. Coincidiendo con Rangel-Salazar et al. (2009), esta cualidad del paisaje de servir como refugio para la avifauna es propiciada por la complejidad estructural que otorga la presencia de arbolado abundante en todas las unidades del paisaje. Esta condición del paisaje cumple con algunas de las sugerencias que Martínez-Ramos \& García-Orth (2007) hacen sobre cómo lograr el manejo ecológicamente sustentable de bosques tropicales que supone combinar reservas de estos hábitats con matrices manejadas incluyendo el componente arbóreo en los pastizales y la sucesión vegetal arbórea en zonas de manejo agrícola.

La semejanza entre los ensambles de la matriz agropecuaria y del bosque dentro del paisaje bajo estudio resulta notable; no obstante, si la reserva de bosque desapareciera seguramente se extinguirían las especies restringidas a ese tipo de hábitat en la zona, especialmente las de estrecho rango de distribución (Johns 1991, ArriagaWeiss et al. 2008, Sodhi et al. 2009), quedando solo la fracción de avifauna más adaptada a las áreas ocupadas por la agricultura y la ganadería. La calidad ecológica del paisaje está asociada indisolublemente a la conservación de una reserva de bosque continuo dentro del territorio manejado por la comunidad local.

\section{CONCLUSIONES}

La avifauna es impactada por las prácticas de manejo que configuran la matriz del paisaje del ejido, modificando la composición del ensamble de aves nativa del bosque tropical perennifolio. No obstante, la combinación de remanentes de bosque con una matriz heterogénea, enriquecida por vegetación secundaria y por potreros con arbolado en línea o disperso, brinda refugio a diferentes especies y gremios de forrajeo nativos de nuestro sitio de estudio que constituyen una ornitofauna notablemente diversa en el sitio de estudio. 
Existen especies indicadoras e incluso familias que permiten la diferenciación de cada condición del hábitat y un alto porcentaje de ellas prefieren elementos de la matriz agropecuaria o del bosque. Las especies del interior del bosque que no estuvieron representadas en la matriz son especies útiles para el monitoreo del estado de conservación de los remanentes del bosque tropical de la zona.

Las tendencias actuales en el ejido, orientadas hacia incrementar el área de potreros y al abandono del sistema de milpa de rotación con periodos mayores a los 30 años representa una amenaza para la estabilidad y permanencia de las comunidades de aves del sistema y en especial para las especies que dependen del bosque maduro pues pueden desaparecer. La mejora de cobertura arbórea en los potreros, rescatar el sistema de milpa con barbecho prolongado y mantener la reserva de bosque tropical perennifolio ejidal representan las mejores opciones para mantener la diversidad de la avifauna en ese paisaje.

Agradecimientos. A los ejidatarios de Niños Héroes de Chapultepec, Tenosique por permitir el acceso a sus parcelas. A Isidro Pérez Hernández por su apoyo en campo. A ECOSUR por las facilidades humanas, científicas, de infraestructura y logísticas. A la DIA-SRE del gobierno de México por la beca 811-06-3-728.5/6457/07 para los estudios de doctorado del primer autor. A UCATSE por favorecer mis estudios en México. Se agradece a dos revisores anónimos que con sus aportes hicieron posible mejorar sustancialmente este artículo.

\section{LITERATURA CITADA}

Almazán-Núñez, R.C., F. Puebla-Olivares \& A. Almazán-Juárez. 2009. Diversidad de aves en bosque de pino-encino del centro de Guerrero, México. Acta Zoológica Mexicana (n.s.), 25: 123-142.

Álvarez-Areces, M. 2009. Patrimonio, cultura y paisaje, recursos para una economía sustentable. Ambienta, 88: 9-19.

Anderson, D. L. 2010. Structure and organization of canopy bird assemblages in lowland neotropical rainforests. Dissertation Doctor in Philosophy, Department of Biological Sciences, Louisiana State University and Agricultural and Mechanical College. $116 \mathrm{pp}$.

Andrade, G. I. \& H. Rubio-Torgler. 1994. Sustainable use of the tropical rain forest: evidence from the avifauna in a shifting-cultivation habitat mosaic in the Colombian Amazon. Conservation Biology, 8: 545-554.

Arriaga-Weiss, S., S. Calmé \& C. Kampichler. 2008. Bird communities in rainforest fragment: guild responses to habitat variables in Tabasco, Mexico. Biodiversity Conservation, 17: 173-190.

Atauri, J. A. \& J. V. De Lucio. 2001. The role of landscape structure in species richness distribution of birds, amphibians, reptiles and lepidopterans in Mediterranean landscapes. Landscape Ecology, 16: 147-159.

Beier, P. \& R. F. Noss. 1998. Do habitat corridors provide connectivity? Conservation Biology, 12: 1241-1252.

Bell, S., M. Fonseca \& L. Motten. 1997. Linking restoration and landscape ecology. Restoration Ecology, 5: 318-323.

Bibby, C., M. Jones \& S. Marsden. 2000. Expedition field techniques: bird surveys. BirdLife International, Cambridge UK. 137 pp. 
Bildstein, K. L. \& J. I. Zalles. 2001. Raptor migration along the Mesoamerican land corridor. Pp. 119141. In: Bildstein \& Klem (Eds.). Hawkwatching in the Americas. Philadelphia, USA.

Bojorges-Baños, J. C. \& L. López-Mata. 2006. Asociación de la riqueza y diversidad de especies de aves y estructura de la vegetación en una selva mediana subperennifolia en el centro de Veracruz, México. Revista Mexicana de Biodiversidad, 77: 235-249.

Bojorjes-Baños, J. C. \& L. López-Mata. 2001. Abundancia y distribución temporal de aves en una selva mediana subperennifolia en el centro de Veracruz, México. Anales del Instituto de Biología, 72: 259-283.

Box, G. \& D. Cox. 1964. An analysis of transformations. Journal of Research of the Statistical Society, 26: 211-243.

Cárdenas, G., C. A. Harvey, M. Ibrahim \& B. Finegan. 2003. Diversidad y riqueza de aves en diferentes hábitats en un paisaje fragmentado en Cañas, Costa Rica. Agroforestería de las Américas, 10: 78-85.

Castelleta, M., N. S. Sodhi \& R. Subaraj. 2000. Heavy extinctions of forest avifauna in Singapore: Lessons for biodiversity conservation in southeast Asia. Conservation Biology, 14: 1870-1880.

Castellón, T. D. \& K. E. Sieving. 2006. An experimental test of matrix permeability and corridor use by an endemic understory bird. Conservation Biology, 20: 135-145.

Cerezo, A., C. S. Robbins \& B. Dowell. 2009. Uso de hábitats modificados por aves dependientes de bosque tropical en la región caribeña de Guatemala. Revista de Biología Tropical, 57: 401-419.

Clarke, K.R., \& R.N. Gorley. 2006. PRIMER v6: User Manual/Tutorial. PRIMER-E, Plymouth.

Clench, H. 1979. To make regional lists of butterflies some thoughts. Lepidopterists' Society, 33: 216231.

Colwell, R. K. 2006. EstimateS: Statistical estimation of species richness and shared species from samples. Version 7.5.2. User's guide and application published at: http://viceroy.eeb.uconn.edu/estimates.

Connell, J. H. 1978. Diversity in tropical rain forest and coral reefs. Science, 199: 1302-1310.

Da Silva, J. M. C., C. Uhl \& G. Murray. 1996. Plant succession, landscape management, and the ecology of frugivorous birds in abandoned Amazonian pastures. Conservation Biology, 10: 491-503.

Daily, G. C., P. R. Ehrlich \& A. Sánchez-Azofeifa. 2001. Countryside biogeography: Use of humandominated habitats by the avifauna of Southern Costa Rica. Ecological Applications, 11: 1-13.

Develey, P. F. \& P. C. Stouffer. 2001. Effects of roads on movements by understory birds in mixedspecies flocks in Central Amazonian Brazil. Conservation Biology, 15: 1416-1422.

DOF (Diario Oficial de la Federación). 2002. Norma oficial mexicana NOM-059-SEMARNAT-2001 protección ambiental- Especies nativas de México de flora y fauna silvestres-Categorías de riesgo y especificaciones para su inclusión, exclusión o cambio- Lista de especies en riesgo. 6 de Marzo de 2002. 153 p. www.seduma.yucatan.gob.mx/./NOMs/NOM-059-SEMARNAT-2001.pdf

DOF (Diario Oficial de la Federación). 2008. Decreto por el que se declara Área Natural Protegida con la categoría de área de protección de flora y fauna, la región conocida como Cañón del Usumacinta, localizada en el Municipio de Tenosique, en el Estado de Tabasco. 22 de Septiembre de 2008. 16 p. http://vlex.com.mx/vid/categoria-flora-fauna-usumacinta-tenosique-42835362

Driscoll, D. A. 2005. Is the matrix a sea? Habitat specificity in a naturally fragmented landscape. Ecological Entomology, 30: 8-16.

Estrada A., R. Coates-Estrada \& D. A. Merrit. 1997. Anthropogenic landscape changes and avian diversity at Los Tuxtlas, México. Biodiversity and Conservation 6: 19-43.

Farina, A. 1997. Landscape structure and breeding bird distribution in a sub-Mediterranean agroecosystem. Landscape Ecology, 12: 365-378. 
Filloy, J. \& M. I. Bellocq. 2007. Respuesta de las aves rapaces al uso de la tierra: un enfoque regional. Hornero, 22: 131-140.

Fleishman, E., C. J. Betrus, R. B. Blair, R. MacNally \& D. D. Murphy. 2002. Nestedness analysis and conservation planning: the importance of place, environment, and life history across taxonomic groups. Oecología, 113: 78-89.

Fletcher, R. J. \& R. L. Hutto. 2008. Partitioning the multi-scale effects of human activity on the occurrence of riparian forest birds. Landscape Ecology, 23: 727-739.

Francesconi, W. 2006. Bird composition in living fences: Potential of living fences to connect the fragmented landscape in Esparza, Costa Rica. Spring, 25: 38-44.

Gillespie, T. \& H. Walter. 2001. Distribution of bird species richness at a regional scale in tropical dry forest of Central America. Journal of Biogeography, 28: 651-662.

Gillespie, T. 2002. Latitudinal extent and natural history characteristics of birds in Nicaragua. Global Ecology \& Biogeography, 11: 411-417.

Gobierno del Estado de Tabasco. 1997. Municipio de Tenosique. Secretaria de Desarrollo Social y Protección Ambiental, Gobierno del Estado de Tabasco. Villahermosa, Tabasco, México.

González-García, F. 2002. Biblioteca de Sonidos de Aves de México. Instituto de Ecología, Xalapa, Veracruz, México. (Consultado en línea: 27 de marzo de 2010, http://www1.inecol.edu.mx/sonidos/menu.htm)

González-Valdivia, N. A. 2010. Conservación de biodiversidad y sustentabilidad en un paisaje cultural del sureste mexicano. Tesis de Doctorado en Ciencias, El Colegio de la Frontera Sur, Tabasco, México. 236 pp.

Gray, M. A., S. L. Baldauf, P. J. Mayhew \& J. K. Hill. 2007. The response of avian feeding guilds to tropical forest disturbance. Conservation Biology, 21: 133-141.

Guevara, S. \& J. Laborde. 1993. Monitoring seed dispersal at isolated standing trees in tropical pastures: consequences for local species availability. Vegetatio, 107/108: 319-338.

Hammer, Ø., D. Harper \& P. Ryan. 2001. Past: Paleontological Statistics Software Package for education and data analysis. Paleontología Electrónica, 4: 9 p. http://palaeo-electronica.org/2001_1/ past/issue1_01.htm.

Harvey, C. \& J. González-Villalobos. 2007. Agroforestry systems conserve species-rich but modified assemblages of tropical birds and bats. Biodiversity Conservation, 16: 2257-2292.

Harvey, C. A., C. Villanueva, J. Villacis, M. Chacón, D. Muñoz, M. López, M. Ibrahim, R. Gómez, R. Taylor, J. Martínez, A. Navas, J. Saenz, D. Sánchez, A. Medina, S. Vilchez, B. Hernández, A. Perez, F. Ruiz, F. López, I. Lang \& F. L. Sinclair 2005. Contribution of live fences to the ecological integrity of agricultural landscapes. Agriculture, Ecosystems and Environment, 111: 200230.

Herrando-Pérez, S. 2002. Manual de ecología matemática. Un enfoque práctico al análisis multivariado (PCA, CLUSTER y MDS) para detectar patrones en ecología. Manual de Referencia, $2^{\mathrm{a}}$ Ed. ECOSUR, Chetumal, Quintana Roo. 60 pp.

Higgs, E. 2003. Historicity and reference in ecological restoration. Pp. 131-177. In Nature for design: People, natural processes and ecological restoration. Massachusetts Institute of Technology, Cambridge.

Hill, J. K. \& K. C. Hamer. 2004. Determining impacts of habitat modification on diversity of tropical forest fauna: The importance of spatial scale. Journal of Applied Ecology, 41: 744-754.

Howell, S. \& S. Webb. 1995. A guide to the birds of México and northern Central America. Oxford University Press Inc., Oxford, Nueva York, EU. 851 pp.

Hueck-Espino, J. 2005. La fauna de la Reserva Biológica Indio Maíz. IBESo/FARENA, Universidad Nacional Agraria, Managua, Nicaragua. 33 pp. 
Hutto, R. L., S. M. Pletschet \& P. Hendricks. 1986. A fixed-radius point count method for nonbreeding and breeding season use. The Auk 103: 593-602.

Ibarra, A. C., S. Arriaga-Weiss \& A. Estrada. 2001. Avifauna asociada a dos cacaotales tradicionales en la región de la Chontalpa, Tabasco, México. Universidad y Ciencia, 17: 101-112.

Isaac-Márquez, F., B. de Jong, A. Eastmond, S. Ochoa-Gaona y S. Hernández. 2005. Estrategias productivas campesinas: un análisis de los factores condicionantes del uso de suelo en el Oriente de Tabasco, México. Universidad y Ciencia, 21: 56-72.

Johns, A. D. 1991. Responses of Amazonian rain forest birds to habitat modification. Journal of Tropical Ecology, 7: 417-437.

Kupfer, J., G. Malanson \& S. Franklin. 2006. Not seeing the ocean for the islands: the mediating influence of matrix-based processes on forest fragmentation effects. Global Ecology and Biogeography, 15: 8-20.

Lang, I., L. H. M. Gormley, C. A. Harvey \& F. L. Sinclair. 2003. Composición de la comunidad de aves en cercas viva de Río Frío, Costa Rica. Agroforestería de las Américas, 10: 86-92.

Lehouk, V., P. Spanhove, C. Vangestel, N. J. Cordeiro \& L. Lens. 2009. Does landscape structure affect resource tracking by avian frugivores in a fragmented afrotropical forest? Ecography, 32: 789-799.

Lepš, J. \& P. Šmilauer. 2005. Multivariate analysis of ecological data using CANOCO. Cambridge University Press., UK. 269 pp.

Luginbühl, Y. 1998. Synthèse et conclusions. Identification des paysages méditerranéens. In: Arias Abellón \& Forneau (Eds.). El paisaje Mediterráneo. Universidad de Granada, Granada, Andalucía, España. 349 pp.

MacNally, R., A. F. Bennett, G. W. Brown, L. F. Lumsden, A. Yen, S. Hinkley, P. Lillywhite \& D. Ward. 2002. How well do ecosystem-based planning units represent different components of biodiversity?. Ecological Applications, 12: 900-912.

Martin, E. A., L. Ratsimisetra, F. Laloë \& S. M. Carrière. 2009. Conservation value for birds of traditionally managed isolated trees in an agricultural landscape of Madagascar. Biodiversity and Conservation. Doi. 10.1007/s10531-009-9671-x.

Martínez-Ramos, M. \& X. García-Orth. 2007. Sucesión ecológica y restauración de las selvas húmedas. Boletín de la Sociedad Botánica de México, 80: 69-84.

Morrison, E. 1999. Introduction to SPSS. Center for Social Science Computation and Research, University of Washington, USA. 14 pp.

Ochoa-Gaona, S., I. Pérez-Hernández \& B. De Jong. 2008. Fenología reproductiva de las especies arbóreas del bosque tropical de Tenosique, Tabasco, México. Revista de Biología Tropical, 56: 657-673.

Olson, D. M., E. Dinerstein, G. V. N. Powell \& E. D. Wikramanayake. 2002. Conservation biology for the biodiversity crisis. Conservation Biology, 16: 1-3.

Patten, A. M., H. Gómez \& B. Smith-Patten. 2010. Long-term changes in the bird community of Palenque, Chiapas, in response to rainforest loss. Biodiversity and Conservation, 19: 21-36.

Pérez, M., M. Sotelo, I. Siria, R. Alkemade \& L. Aburto. 2007. Developing a species based model for biodiversity assessment in an agricultural landscape in Nicaragua. Gaia, 8: 1-54.

Perfecto, I. \& J. Vandermeer. 2002. Quality of agroecological matrix in a tropical montane landscape: ants in coffee plantations in southern Mexico. Conservation Biology, 16: 174-182.

Perfecto, I. \& J. Vandermeer. 2008. Biodiversity conservation in tropical agroecosystems: A new conservation paradigm. Annual New York Academic Science, 1134: 173-200.

Pulido-Santacruz, P. \& L. M. Renjifo. 2011. Live fences as tools for biodiversity conservation: a study case with birds and plants. Agroforestry Systems 81:15-30. 
Ramírez, A. 2006. Ecología: Métodos de muestreo y análisis de poblaciones y comunidades. Editorial Pontificia Universidad Javeriana, Bogotá, Colombia. 273 pp.

Ramírez-Albores, J. E. 2010. Diversidad de aves de hábitats naturales y modificados en un paisaje de la Depresión Central de Chiapas, México. Revista de Biología Tropical, 58: 511-528.

Rangel-Salazar, J.L., P. L. Enríquez \& E. C. Sántiz-López. 2009. Variación de la diversidad de aves de sotobosque en el Parque Nacional Lagos de Montebello, Chiapas, México. Acta Zoológica Mexicana, 25: 479-495.

Rich, P. M., J. Wood, D. A. Vieglais, K. Burek \& N. Webb. 1999. Hemiview User Manual. Delta-T Devices, Ltd. http://www.delta-t.co.uk/support-article.html?article = faq2005100703399

Robinson, W. D. 2001. Changes in abundance of birds in a neotropical forest fragment over 25 years: a review. Animal Biodiversity and Conservation, 24: 51-65.

Rossignol, M., L. Rossignol, R. Oldeman \& S. Benzine-Tizroutine. 1998. Struggle of life or the natural history of stress and adaptation. Treebook 1, Wageningen, Países Bajos. 237 pp.

Schröth, G. \& C. Harvey. 2007. Biodiversity conservation in cocoa production landscapes: an overview. Biodiversity Conservation, 16: 2237-2244.

Shankar-Raman, T. R., G. S. Rawat \& J. T. Johnsingh. 1998. Recovery of tropical rainforest avifauna in relation to vegetation succession following shifting cultivation in Mizoram, north-east India. Journal of Applied Ecology, 35: 214-231.

Sodhi, N.S., T. Ming-Lee, L. Pin-Koh \& B.W. Brook. 2009. A meta-analysis of the impact of anthropogenic forest disturbance on southeast Asia's Biotas. Biotropica, 41: 103-109.

Stouffer, P., R.O. Bierregaard, C. Strong. \& T. E. Lovejoy. 2006. Long-term landscape change and bird abundance in Amazonian rainforest fragments. Conservation Biology, 20: 1212-1223.

Tews, J., U. Brose, V. Grimm, K. Tielbörger, M.C. Wichmann, M. Schwager \& F. Jeltsch. 2004. Animal species diversity driven by habitat heterogeneity/diversity: the importance of keystone structures. Journal of Biogeography, 31: 79-92.

Turner, M., R. Gardner. \& R. O’Neill. 2001. Landscape ecology in theory and practice: pattern and process. Springer-Verlag, New York, EEUU. 401 pp.

Van Perlo, B. 2006. Birds of Mexico and Central America. Illustrated Checklist. Princeton University Press. New Jersey, USA. 336 pp.

Vandermeer, J. \& I. Perfecto. 2007. The agricultural matrix and a future paradigm for conservation. Conservation Biology, 21: 274-277.

Vázquez-Pérez, J. R., Enríquez, P. L. \& Rangel-Salazar, J. L. 2009. Diversidad de aves rapaces diurnas en la Reserva de la Biósfera Selva El Ocote, Chiapas, México. Revista Mexicana de Biodiversidad, 80: 203-209.

Vielliard, J. M. E. 2000. Bird community as an indicator of biodiversity: results from quantitative surveys in Brazil. Anais da Academia Brasileira de Ciências, 72: 323-330.

Watling, J. I. \& M. A. Donnelly. 2006. Fragments as islands: a synthesis of faunal responses to habitat patchiness. Conservation Biology, 20: 1016-1025.

Zonneveld, I. 1989. The land unit: A fundamental concept in landscape ecology, and its application. Landscape Ecology, 3: 67-83.

Zuur, A., E. Ieno \& G. Smith. 2007. Analyzing ecological data. Pp. 163-179. In: M. Gail, K. Krickeberg, J. Samet, A. Tsiatis and W. Wong, Series (Eds.). Statistics for biology and health. Springer Science + BusinessMedia, LLC, 233 Spring Street, New York, USA. 


\section{ANEXO 1. AVIFAUNA REGISTRADA PARA EL PAISAJE DEL EJIDO NHC EN DOS ESTACIONES DURANTE EL AÑO 2008.}

Claves:

$\mathbf{R}=$ Estatus de residencia: $\mathrm{RES}=$ Residente, MIG $=$ Migrante, MIE $=$ Migrante local estacional

$\mathbf{G}=$ Gremio: $\mathrm{CA}=$ Cazador acuático, $\mathrm{CAG}=$ Cazador asociado a ganado, $\mathrm{CAR}=$ Carroñero, $\mathrm{CC}=$ Cazador que captura presas sobre corteza o en epifitas, $\mathrm{CM}=$ Cazador que captura presa dentro de la madera (carpintero), $\mathrm{GFA}=$ Granívoro-frugívoro arbóreo, GFT = Granívoro-frugívoro terrestre, IAM = Insectívoro pequeño que caza sin percha en el estrato alto y medio, IBH = Insectívoro pequeño que caza sin percha en el estrato arbustivo bajo y herbáceo, IG = Insectívoro grande cazador bajo el dosel desde percha ( $\geq 15 \mathrm{~cm}$ longitud), IP = Insectívoro pequeño cazador bajo el dosel desde percha ( $15 \mathrm{~cm}$ longitud), IS = Insectívoro en el suelo, IV = Insectívoro que caza al vuelo sobre el dosel, $\mathrm{N}=$ Nectarívoro, $\mathrm{OA}$ = Omnívoros arbóreo, $\mathrm{OM}$ = Omnívoros, $\mathrm{RD}=$ Rapaz diurna.

$\mathbf{E}=$ Ecomosaicos en que se encontró especie: $\mathrm{MA}=$ Matriz agropecuaria, $\mathrm{RE}=$ Bosque tropical perennifolio, $\mathrm{C}=$ en ambos.

$\mathbf{M}=$ Estación climática en que se registró: $\mathrm{Se}=\mathrm{Seca}, \mathrm{Ll}=$ Lluviosa, $\mathrm{A}=$ Ambas.

NOM = indica especies citadas en la NOM-059-ECOL-2001 (DOF 2002).

\begin{tabular}{|c|c|c|c|c|c|}
\hline Familia & Especie & $\mathrm{R}$ & G & $\mathrm{E}$ & M \\
\hline \multirow[t]{3}{*}{ Ardeidae } & Ardea herodias Linnaeus & RES & CA & MA & Se \\
\hline & Bubulcus ibis Linnaeus & RES & CAG & MA & A \\
\hline & Egretta caerulea Linnaeus & RES & CA & MA & Se \\
\hline Aramidae & Aramus guarauna Linnaeus & RES & CA & MA & A \\
\hline Anatidae & Dendrocygna autumnalis Linnaeus & MIE & CA & MA & Se \\
\hline \multirow[t]{7}{*}{ Accipitridae } & Accipiter bicolor Vieillot ${ }^{\mathrm{NOM}}$ & RES & $\mathrm{RD}$ & $\mathrm{RE}$ & Se \\
\hline & Asturina nitida (Latham) & RES & $\mathrm{RD}$ & $\mathrm{RE}$ & Se \\
\hline & Buteo magnirostris Gmelin & RES & $\mathrm{RD}$ & MA & Se \\
\hline & Buteogallus urubitinga Gmelin ${ }^{\mathrm{NOM}}$ & RES & $\mathrm{RD}$ & MA & Se \\
\hline & Chondrohierax uncinatus Temminck ${ }^{\mathrm{NOM}}$ & RES & $\mathrm{RD}$ & MA & A \\
\hline & Elanoides forficatus (Linnaeus) ${ }^{\mathrm{NOM}}$ & MIE & $\mathrm{RD}$ & $\mathrm{RE}$ & Se \\
\hline & Leucopternis albicollis Latham ${ }^{\mathrm{NOM}}$ & RES & $\mathrm{RD}$ & $\mathrm{C}$ & Se \\
\hline \multirow[t]{2}{*}{ Falconidae } & Falco peregrinus Tunstall & MIG & $\mathrm{RD}$ & MA & Se \\
\hline & Herpetotheres cachinnans Linnaeus & RES & $\mathrm{RD}$ & MA & $\mathrm{Ll}$ \\
\hline \multirow[t]{2}{*}{ Cathartidae } & Coragyps atratus Bechstein & RES & CAR & MA & A \\
\hline & Sarcorhampus papa Linnaeus ${ }^{\mathrm{NOM}}$ & RES & CAR & MA & $\mathrm{Ll}$ \\
\hline \multirow[t]{2}{*}{ Cracidae } & Crax rubra Linnaeus ${ }^{\mathrm{NOM}}$ & RES & GFT & $\mathrm{RE}$ & A \\
\hline & Ortalis vetula Wagler & RES & GFT & $\mathrm{C}$ & A \\
\hline \multirow[t]{3}{*}{ Tinamidae } & Crypturellus boucardi Sclater ${ }^{\mathrm{NOM}}$ & RES & GFT & $\mathrm{C}$ & A \\
\hline & Crypturellus soui Hermann ${ }^{\mathrm{NOM}}$ & RES & GFT & $\mathrm{C}$ & A \\
\hline & Tinamus major Gmelin ${ }^{\mathrm{NOM}}$ & RES & GFT & $\mathrm{RE}$ & A \\
\hline \multirow[t]{2}{*}{ Odontophoridae } & Colinus nigrogularis Gould & RES & GFT & $\mathrm{RE}$ & $\mathrm{Ll}$ \\
\hline & Colinus virginianus Linnaeus & RES & GFT & MA & A \\
\hline
\end{tabular}




\begin{tabular}{|c|c|c|c|c|c|}
\hline Familia & Especie & $\mathrm{R}$ & G & $\mathrm{E}$ & M \\
\hline & Dactylortix thoracicus Gambel ${ }^{\mathrm{NOM}}$ & RES & GFT & $\mathrm{RE}$ & $\mathrm{Ll}$ \\
\hline & Odontophorus guttatus Gould NOM & RES & GFT & $\mathrm{C}$ & A \\
\hline Eurypygidae & Eurypyga helias Pallas ${ }^{\mathrm{NOM}}$ & MIG & CA & MA & $\mathrm{Ll}$ \\
\hline \multirow[t]{10}{*}{ Columbidae } & Claravis pretiosa (Ferrari-Pérez) & RES & GFT & MA & A \\
\hline & Columba cayennensis Bonnaterre & RES & GFT & $\mathrm{C}$ & A \\
\hline & Columba flavirostris Wagler & RES & GFT & $\mathrm{C}$ & A \\
\hline & Columba nigrirostris Sclater ${ }^{\mathrm{NOM}}$ & RES & GFT & $\mathrm{C}$ & A \\
\hline & Columbina minuta Linnaeus & RES & GFT & MA & A \\
\hline & Geotrygon montana Linnaeus & MIE & GFT & $\mathrm{C}$ & Se \\
\hline & Leptotila cassini Lawrence & RES & GFT & $\mathrm{C}$ & A \\
\hline & Leptotila plumbeiceps Sclater \& Salvin & RES & GFT & $\mathrm{C}$ & Se \\
\hline & Leptotila verreauxi Bonaparte & RES & GFT & $\mathrm{C}$ & A \\
\hline & Zenaida asiatica Linnaeus & RES & GFT & MA & Se \\
\hline \multirow[t]{5}{*}{ Psittacidae } & Amazona autumnalis Linnaeus & MIE & GFA & $\mathrm{RE}$ & Se \\
\hline & Amazona farinosa Boddaert ${ }^{\mathrm{NOM}}$ & MIE & GFA & $\mathrm{C}$ & A \\
\hline & Aratinga holochlora Sclater ${ }^{\mathrm{NOM}}$ & MIE & GFA & $\mathrm{C}$ & A \\
\hline & Aratinga nana Vigors $^{\mathrm{NOM}}$ & MIE & GFA & $\mathrm{C}$ & Se \\
\hline & Pionus senilis Spix ${ }^{\mathrm{NOM}}$ & MIE & GFA & $\mathrm{C}$ & Se \\
\hline \multirow[t]{4}{*}{ Cuculidae } & Crotophaga sulcirostris Swainson & RES & CAG & MA & A \\
\hline & Dromococcyx phasianellus Spix & RES & $\mathrm{OM}$ & $\mathrm{RE}$ & $\mathrm{Ll}$ \\
\hline & Morococcyx erythropygus Lesson & RES & $\mathrm{OM}$ & MA & $\mathrm{Ll}$ \\
\hline & Piaya cayana Linnaeus & RES & $\mathrm{OM}$ & $\mathrm{RE}$ & Se \\
\hline Apodidae & Chaetura vauxii Townsend & RES & IV & MA & A \\
\hline \multirow[t]{12}{*}{ Trochilidae } & Amazilia cyanocephala Lesson & MIE & $\mathrm{N}$ & $\mathrm{RE}$ & Se \\
\hline & Amazilia tzacatl De la Llave & RES & $\mathrm{N}$ & $\mathrm{C}$ & A \\
\hline & Amazilia yucatanensi Cabot & MIE & $\mathrm{N}$ & $\mathrm{RE}$ & Se \\
\hline & Anthracothorax prevostii Lesson & MIE & $\mathrm{N}$ & $\mathrm{C}$ & A \\
\hline & Campylopterus curvipennis Deppe & MIE & $\mathrm{N}$ & $\mathrm{C}$ & Se \\
\hline & Campylopterus rufus Lesson ${ }^{\mathrm{NOM}}$ & MIE & $\mathrm{N}$ & $\mathrm{RE}$ & Se \\
\hline & Chlorostilbon canivetii Lesson & MIE & $\mathrm{N}$ & $\mathrm{C}$ & A \\
\hline & Chlorostilbon salvini Cabanis \& Heine & MIE & $\mathrm{N}$ & $\mathrm{C}$ & Se \\
\hline & Eupherusa eximia DeLattre & RES & $\mathrm{N}$ & $\mathrm{C}$ & A \\
\hline & Heliomaster longirostris Audebert \& Vieillot ${ }^{\mathrm{NOM}}$ & MIE & $\mathrm{N}$ & $\mathrm{C}$ & Se \\
\hline & Hylocharis eliciae Bourcier \& Mulsant & MIE & $\mathrm{N}$ & $\mathrm{RE}$ & Se \\
\hline & Lampornis amethystinus Swainson & MIE & $\mathrm{N}$ & $\mathrm{C}$ & Se \\
\hline
\end{tabular}




\begin{tabular}{|c|c|c|c|c|c|}
\hline Familia & Especie & $\mathrm{R}$ & G & $\mathrm{E}$ & M \\
\hline & Phaethornis longirostris Delattre & MIE & $\mathrm{N}$ & $\mathrm{RE}$ & Se \\
\hline & Phaethornis mexicanus Hartert & MIE & $\mathrm{N}$ & $\mathrm{RE}$ & Se \\
\hline & Phaethornis striigularis Gould & MIE & $\mathrm{N}$ & MA & Se \\
\hline & Threnetes ruckeri Bourcier & MIE & $\mathrm{N}$ & MA & Se \\
\hline \multirow[t]{4}{*}{ Trogonidae } & Trogon citreolus Gould & RES & OA & $\mathrm{C}$ & A \\
\hline & Trogon massena Gould ${ }^{\mathrm{NOM}}$ & RES & OA & $\mathrm{C}$ & A \\
\hline & Trogon melanocephalus Gould & RES & $\mathrm{OA}$ & $\mathrm{C}$ & A \\
\hline & Trogon violaceus Gmelin & RES & OA & $\mathrm{C}$ & A \\
\hline Ramphastidae & Ramphastos sulfuratus Lesson ${ }^{\mathrm{NOM}}$ & RES & GFA & $\mathrm{C}$ & A \\
\hline \multirow[t]{2}{*}{ Momotidae } & Hylomanes momotula Lichtenstein ${ }^{\mathrm{NOM}}$ & RES & IP & $\mathrm{C}$ & $\mathrm{Ll}$ \\
\hline & Momotus momota Linnaeus & RES & IG & $\mathrm{C}$ & A \\
\hline Galbulidae & Galbula ruficauda Cuvier ${ }^{\mathrm{NOM}}$ & RES & IP & $\mathrm{RE}$ & A \\
\hline \multirow[t]{7}{*}{ Picidae } & Campephilus guatemalensis Hartlaub ${ }^{\mathrm{NOM}}$ & RES & $\mathrm{CM}$ & $\mathrm{RE}$ & A \\
\hline & Dryocopus lineatus Linnaeus & RES & $\mathrm{CM}$ & $\mathrm{C}$ & A \\
\hline & Melanerpes aurifrons Wagler & RES & $\mathrm{CM}$ & $\mathrm{C}$ & A \\
\hline & Melanerpes pucherani Malherbe & RES & $\mathrm{CM}$ & MA & $\mathrm{Ll}$ \\
\hline & Picoides scalaris Wagler & RES & $\mathrm{CM}$ & $\mathrm{C}$ & Se \\
\hline & Piculus rubiginosus Swainson & RES & $\mathrm{CM}$ & $\mathrm{C}$ & A \\
\hline & Veniliornis fumigatus D’Orbigny & RES & $\mathrm{CM}$ & $\mathrm{RE}$ & A \\
\hline \multirow[t]{7}{*}{ Dendrocolaptidae } & Dendrocincla anabatina Sclater ${ }^{\mathrm{NOM}}$ & RES & CC & $\mathrm{C}$ & A \\
\hline & Dendrocincla homochroa Sclater & RES & CC & $\mathrm{C}$ & A \\
\hline & Dendrocolaptes sanctithomae Lafresnaye & RES & $\mathrm{CC}$ & $\mathrm{C}$ & A \\
\hline & Glyphorhynchus spyrurus Vieillot ${ }^{\mathrm{NOM}}$ & RES & CC & $\mathrm{C}$ & A \\
\hline & Lepidocolaptes souleyetii Des Murs & RES & CC & $\mathrm{C}$ & A \\
\hline & Sittasomus griseicapillus Vieillot & RES & $\mathrm{CC}$ & $\mathrm{C}$ & A \\
\hline & Xiphorhynchus flavigaster Swainson & RES & CC & $\mathrm{C}$ & A \\
\hline \multirow[t]{4}{*}{ Furnariidae } & Automolus ochrolaemus Tschudi ${ }^{\mathrm{NOM}}$ & RES & IS & $\mathrm{RE}$ & Se \\
\hline & Automolus rubiginosus (Sclater) ${ }^{\mathrm{NOM}}$ & RES & $\mathrm{IBH}$ & $\mathrm{RE}$ & $\mathrm{Ll}$ \\
\hline & Sclerurus guatemalensis (Hartlaub) ${ }^{\mathrm{NOM}}$ & RES & IS & $\mathrm{C}$ & Se \\
\hline & Xenops minutus Sparrman ${ }^{\mathrm{NOM}}$ & RES & CC & $\mathrm{C}$ & A \\
\hline \multirow[t]{4}{*}{ Formicaridae } & Cercomacra tyrannina Sclater & RES & IBH & MA & Se \\
\hline & Dysithamnus mentalis Temminck ${ }^{\mathrm{NOM}}$ & RES & IBH & $\mathrm{RE}$ & Se \\
\hline & Formicarius nigricapillus Ridgway & RES & IS & $\mathrm{RE}$ & Se \\
\hline & Thamnistes anabatinus Sclater \& Salvin ${ }^{\mathrm{NOM}}$ & RES & IBH & $\mathrm{RE}$ & Se \\
\hline Sylviidae & Polioptila plumbea Gmelin ${ }^{\mathrm{NOM}}$ & RES & IAM & $\mathrm{C}$ & A \\
\hline
\end{tabular}




\begin{tabular}{|c|c|c|c|c|c|}
\hline Familia & Especie & $\mathrm{R}$ & G & $\mathrm{E}$ & $\mathrm{M}$ \\
\hline & Ramphocaenus melanurus Vieillot & RES & IBH & $\mathrm{C}$ & A \\
\hline \multirow[t]{34}{*}{ Tyrannidae } & Attila spadiceus Gmelin & RES & IAM & $\mathrm{RE}$ & A \\
\hline & Contopus borealis (Swainson) & MIG & IP & $\mathrm{C}$ & A \\
\hline & Contopus cinereus Spix & MIG & IP & $\mathrm{C}$ & Se \\
\hline & Contopus pertinax Cabanis \& Heine & MIG & IP & $\mathrm{C}$ & Se \\
\hline & Contopus virens Linnaeus & MIG & IP & $\mathrm{C}$ & A \\
\hline & Elaenia flavogaster (Thunberg) & MIG & IP & $\mathrm{C}$ & Se \\
\hline & Empidonax affinis Swainson & MIG & IP & $\mathrm{C}$ & A \\
\hline & Empidonax albigularis Sclater \& Salvin & MIG & IP & $\mathrm{C}$ & Se \\
\hline & Empidonax alnorum Brewster & MIG & IP & $\mathrm{C}$ & Se \\
\hline & Empidonax flavescens Lawrence & MIG & IP & $\mathrm{C}$ & A \\
\hline & Empidonax minimus Baird \& Baird & MIG & IP & $\mathrm{C}$ & A \\
\hline & Empidonax traillii Audubon & MIG & IP & $\mathrm{RE}$ & Se \\
\hline & Empidonax virescens Vieillot & MIG & IP & MA & Se \\
\hline & Mionectes oleagineus Lichtenstein & RES & IAM & $\mathrm{C}$ & Se \\
\hline & Mitrephanes phaeocercus Sclater & RES & IP & $\mathrm{RE}$ & Se \\
\hline & Myiarchus cinerascens Lawrence & MIG & IG & $\mathrm{C}$ & Se \\
\hline & Myiarchus crinitus Linnaeus & MIG & IG & $\mathrm{C}$ & A \\
\hline & Myiarchus nuttingi Ridgway & MIG & IG & MA & Se \\
\hline & Myiarchus tuberculifer d'Orbigny \& Lafresnaye & MIG & IG & MA & Se \\
\hline & Myiarchus tyrannulus Statius Müller & MIG & IG & MA & Se \\
\hline & Myiobius sulphureipygius Sclater & RES & IP & $\mathrm{C}$ & A \\
\hline & Myiodynastes luteiventris Sclater & MIG & IG & MA & A \\
\hline & Myiopagis viridicata Vieillot & RES & IP & $\mathrm{RE}$ & Se \\
\hline & Myiozetetes similis Spix & RES & IG & $\mathrm{C}$ & A \\
\hline & Oncostoma cinereigulare (Sclater) & RES & IP & Ма & Se \\
\hline & Onychorhynchus coronatus Statius Müller ${ }^{\mathrm{NOM}}$ & RES & IP & $\mathrm{RE}$ & A \\
\hline & Ornithion semiflavum Sclater \& Salvin ${ }^{\mathrm{NOM}}$ & RES & IP & $\mathrm{C}$ & Se \\
\hline & Pitangus sulphuratus (Linnaeus) & RES & IG & $\mathrm{C}$ & A \\
\hline & Platyrinchus cancrominus Sclater \& Salvin ${ }^{\mathrm{NOM}}$ & RES & IBH & $\mathrm{C}$ & A \\
\hline & Rhytipterna holerythra (Sclater \& Salvin) & RES & IBH & $\mathrm{C}$ & A \\
\hline & Terenotriccus erythrurus Cabanis ${ }^{\mathrm{NOM}}$ & RES & IP & $\mathrm{RE}$ & Se \\
\hline & Todirostrum cinereum Linnaeus & RES & IBH & MA & Se \\
\hline & Todirostrum sylvia Desmarest & RES & IBH & $\mathrm{C}$ & Se \\
\hline & Tolmomyas sulphurescens Spix & RES & IP & $\mathrm{C}$ & Se \\
\hline
\end{tabular}




\begin{tabular}{|c|c|c|c|c|c|}
\hline Familia & Especie & $\mathrm{R}$ & G & $\mathrm{E}$ & M \\
\hline & Tyrannus couchii S.F. Baird & MIG & IG & MA & Se \\
\hline & Tyrannus forficatus Gmelin & MIG & IG & MA & Se \\
\hline & Tyrannus melancholicus Vieillot & MIG & IG & $\mathrm{C}$ & A \\
\hline & Tyrannus savana Vieillot & MIG & IG & MA & Se \\
\hline & Tyrannus tyrannus (Linnaeus) & MIG & IG & MA & Se \\
\hline & Tyrannus verticalis Say & MIG & IG & MA & A \\
\hline & Zimmerius vilissimus Sclater \& Salvin & RES & IP & $\mathrm{RE}$ & Se \\
\hline \multirow[t]{2}{*}{ Pipridae } & Manacus candei Parzudaky NOM & RES & IAM & $\mathrm{C}$ & Se \\
\hline & Pipra mentalis Sclater & RES & IAM & $\mathrm{C}$ & A \\
\hline \multirow[t]{5}{*}{ Cotingidae } & Lipaugus unirufus Sclater & RES & IBH & $\mathrm{C}$ & A \\
\hline & Pachyramphus aglaiae Lafresnaye & RES & IAM & $\mathrm{C}$ & A \\
\hline & Pachyramphus cinnamomeus Lawrence & RES & IAM & MA & Se \\
\hline & Schiffornis turdinus (Wied) & RES & GFA & $\mathrm{C}$ & A \\
\hline & Tytira semifasciata Spix & RES & GFA & $\mathrm{C}$ & A \\
\hline Corvidae & Cyanocorax morio Wagler & RES & $\mathrm{OM}$ & $\mathrm{C}$ & A \\
\hline \multirow[t]{10}{*}{ Troglodytidae } & Henicorhina leucophrys Tschudi & RES & IS & $\mathrm{RE}$ & Se \\
\hline & Henicorhina leucosticta Cabanis & RES & IS & $\mathrm{C}$ & A \\
\hline & Microcerculus philomela Sclater & RES & IS & $\mathrm{C}$ & Se \\
\hline & Thryothorus (albinucha) ludovicianus Latham & RES & IS & $\mathrm{C}$ & A \\
\hline & Thryothorus maculipectus Lafresnaye & RES & IS & $\mathrm{C}$ & A \\
\hline & Thryothorus modestus Cabanis & RES & IS & $\mathrm{C}$ & A \\
\hline & Thryothorus pleurostictus Sclater & RES & IS & $\mathrm{C}$ & A \\
\hline & Troglodytes aedon Vieillot & RES & IS & $\mathrm{C}$ & A \\
\hline & Troglodytes musculus Vieillot & RES & IS & $\mathrm{C}$ & A \\
\hline & Uropsila leucogastra (Gould) & RES & IBH & $\mathrm{C}$ & A \\
\hline \multirow[t]{7}{*}{ Turdidae } & Catharus fuscescens Stephens & MIG & $\mathrm{IBH}$ & $\mathrm{C}$ & Se \\
\hline & Catharus mexicanus Bonaparte ${ }^{\mathrm{NOM}}$ & MIG & IBH & $\mathrm{C}$ & Se \\
\hline & Catharus minimus Lafresnaye & MIG & $\mathrm{IBH}$ & MA & Se \\
\hline & Catharus(Hylocichla) mustelinus Gmelin & MIG & IBH & $\mathrm{C}$ & Se \\
\hline & Catharus ustulatus Nuttal & MIG & IBH & $\mathrm{C}$ & A \\
\hline & Myadestes occidentalis Stejneger ${ }^{\mathrm{NOM}}$ & MIE & IAM & $\mathrm{RE}$ & Se \\
\hline & Turdus grayi Bonaparte & RES & GFT & $\mathrm{C}$ & A \\
\hline \multirow[t]{3}{*}{ Vireonidae } & Hylophilus ochraceiceps Sclater & RES & IBH & MA & Se \\
\hline & Vireo flavoviridis Cassin & MIG & IAM & $\mathrm{C}$ & A \\
\hline & Vireo gilvus Vieillot & MIG & IAM & $\mathrm{C}$ & Se \\
\hline
\end{tabular}




\begin{tabular}{|c|c|c|c|c|c|}
\hline Familia & Especie & $\mathrm{R}$ & G & $\mathrm{E}$ & $\mathrm{M}$ \\
\hline \multirow{35}{*}{ Emberizidae } & Vireo griseus Boddaert & MIG & IAM & $\mathrm{C}$ & A \\
\hline & Vireo hypochryseus Sclater & MIG & IAM & $\mathrm{C}$ & Se \\
\hline & Vireo leucophrys (Lafresnaye) & MIG & IAM & $\mathrm{C}$ & A \\
\hline & Vireo olivaceus Linnaeus & MIG & IAM & $\mathrm{RE}$ & A \\
\hline & Vireo philadelphicus Cassin & MIG & IAM & $\mathrm{C}$ & Se \\
\hline & Vireo plumbeus Coues & MIG & IAM & $\mathrm{C}$ & A \\
\hline & Vireo solitarius Wilson & MIG & IAM & $\mathrm{C}$ & Se \\
\hline & Aimophila rufescens Swainson & RES & GFT & MA & Se \\
\hline & Arremonops chloronotus Salvin & RES & $\mathrm{IBH}$ & $\mathrm{C}$ & Se \\
\hline & Basileuterus culicivorus Deppe & RES & IBH & $\mathrm{C}$ & Se \\
\hline & Basileuterus rufifrons Swainson & RES & IBH & $\mathrm{C}$ & Se \\
\hline & Chlorophanes spiza Linnaeus & RES & $\mathrm{N}$ & $\mathrm{C}$ & A \\
\hline & Cyanerpes cyaneus Linnaeus & RES & $\mathrm{N}$ & $\mathrm{C}$ & Se \\
\hline & Cyanocompsa cyanoides Lafresnaye & RES & GFA & $\mathrm{C}$ & A \\
\hline & Dendroica cerulea Wilson & MIG & IAM & $\mathrm{C}$ & A \\
\hline & Dendroica coronata Linnaeus & MIG & IAM & $\mathrm{C}$ & Se \\
\hline & Dendroica dominica Linnaeus & MIG & IAM & $\mathrm{RE}$ & Se \\
\hline & Dendroica fusca Statius Müller & MIG & IAM & $\mathrm{C}$ & A \\
\hline & Dendroica magnolia Wilson & MIG & IAM & $\mathrm{C}$ & Se \\
\hline & Dendroica petechia Linnaeus & MIG & IAM & $\mathrm{C}$ & A \\
\hline & Dendroica virens Gmelin & MIG & IAM & $\mathrm{RE}$ & Se \\
\hline & Euphonia gouldi Sclater ${ }^{\mathrm{NOM}}$ & RES & IAM & $\mathrm{RE}$ & Se \\
\hline & Euphonia hirundinacea Bonaparte & RES & IAM & $\mathrm{C}$ & A \\
\hline & Granatellus sallaei Bonaparte & RES & IAM & $\mathrm{RE}$ & A \\
\hline & Habia rubica Vieillot & MIG & IBH & $\mathrm{RE}$ & A \\
\hline & Helmitheros vermivorus Gmelin & MIG & IS & $\mathrm{C}$ & Se \\
\hline & Lanio aurantius Lafresnaye ${ }^{\mathrm{NOM}}$ & RES & IAM & $\mathrm{RE}$ & Se \\
\hline & Mniotilta varia Linnaeus & MIG & IAM & $\mathrm{C}$ & A \\
\hline & Oporornis philadelphia Wilson & MIG & IBH & $\mathrm{C}$ & Se \\
\hline & Parula americana Linnaeus & MIG & IBH & $\mathrm{C}$ & Se \\
\hline & Parula pitiayumi Vieillot & MIG & IBH & $\mathrm{C}$ & A \\
\hline & Pheutictus ludovicianus Linnaeus & MIG & GFA & $\mathrm{RE}$ & Se \\
\hline & Piranga rubra Linnaeus & MIG & IAM & $\mathrm{C}$ & Se \\
\hline & Ramphocelus passerinii Bonaparte & RES & IBH & $\mathrm{C}$ & A \\
\hline & Ramphocelus sanguinolentus Lesson & RES & IBH & MA & Se \\
\hline
\end{tabular}




\begin{tabular}{llcccc}
\hline \multicolumn{1}{c}{ Eamilia } & \multicolumn{1}{c}{ Especie } & R & G & E & M \\
\hline \multirow{4}{*}{ Saltator atriceps Lesson } & RES & GFA & C & A \\
& Saltator caerulescens Vieillot & RES & GFA & RE & Se \\
& Setophaga ruticilla (Linnaeus) & MIG & IAM & C & A \\
& Sporophila americana Gmelin & RES & GFT & MA & A \\
& Sporophila torqueola Bonaparte & RES & GFT & MA & A \\
& Tachyphonus delatrii Lafresnaye & MIE & IAM & RE & Se \\
& Thraupis abbas Deppe & RES & GFA & MA & A \\
& Thraupis episcopus Linnaeus & RES & GFA & MA & A \\
& Vermivora peregrina (Wilson) & MIG & IAM & C & A \\
& Vermivora pinus (Linnaeus) & MIG & IAM & C & Se \\
& Vermivora (Parula) superciliosa (Hartlaub) & MIG & IAM & C & Se \\
& Vermivora virginiae (Baird) & MIG & IAM & C & Se \\
& Wilsonia canadensis Linnaeus & MIG & IAM & C & Se \\
& Carduelis psaltria Say & MIG & IAM & C & Se \\
& Dives dives Deppe & RES & GFT & MA & A \\
& Icterus galbula Linnaeus & MIG & GFA & RE & A \\
& Icterus mesomelas Wagler & RES & GFA & MA & A \\
& Icterus spurius Linnaeus & MIG & GFA & C & A \\
& Psarocolius montezuma Lesson ${ }^{\text {NOM }}$ & RES & GFA & C & A \\
& Quiscalus mexicanus Gmelin & RES & OM & MA & A
\end{tabular}

